\title{
Weighing simulated galaxy clusters using lensing and X-ray
}

\author{
M. Meneghetti ${ }^{1,2,5}$, E. Rasia ${ }^{3, \star}$, J. Merten ${ }^{1,5}$, F. Bellagamba ${ }^{7}$, S. Ettori ${ }^{1,2}$, P. Mazzotta $^{4}$, K. Dolag $^{6}$, and S. Marri ${ }^{1}$ \\ 1 INAF-Osservatorio Astronomico di Bologna, via Ranzani 1, 40127 Bologna, Italy \\ e-mail: massimo.meneghetti@oabo.inaf.it \\ 2 INFN-National Institute for Nuclear Physics, Sezione di Bologna, Viale Berti Pichat 6/2, 40127 Bologna, Italy \\ 3 Department of Physics, University of Michigan, 450 Church St., Ann Arbor, MI 48109-1120, USA \\ 4 Dipartimento di Fisica, Università “Tor Vergata”, via della Ricerca Scientifica 1, 00133 Roma, Italy \\ 5 Institut für Theoretische Astrophysik, Zentrum für Astronomie der Universität Heidelberg, Albert Überle Str. 2, 69120 Heidelberg, \\ Germany \\ ${ }^{6}$ Max-Planck-Institute für Astrophysik, PO Box 1317, 85741 Garching b. München, Germany \\ 7 Dipartimento di Astronomia, Università di Bologna, Via Ranzani 1, 40127 Bologna, Italy
}

Received 1 September 2009 / Accepted 1 December 2009

\begin{abstract}
Context. Among the methods employed to measure the mass of galaxy clusters, the techniques based on lensing and X-ray analyses are perhaps the most widely used; however, the comparison between these mass estimates is often difficult and, in several clusters, the results apparently inconsistent.

Aims. We aim at investigating potential biases in lensing and X-ray methods to measure the cluster mass profiles.

Methods. We performed realistic simulations of lensing and X-ray observations that were subsequently analyzed using observational techniques. The resulting mass estimates were compared with the input models. Three clusters obtained from state-of-the-art hydrodynamical simulations, each of which projected along three independent lines-of-sight, were used for this analysis.

Results. We find that strong lensing models can be trusted over a limited region around the cluster core. Extrapolating the strong lensing mass models to outside the Einstein ring can lead to significant biases in the mass estimates, if the BCG is not modeled properly, for example. Weak-lensing mass measurements can be strongly affected by substructures, depending on the method implemented to convert the shear into a mass estimate. Using nonparametric methods which combine weak and strong lensing data, the projected masses within $R_{200}$ can be constrained with a precision of $\sim 10 \%$. Deprojection of lensing masses increases the scatter around the true masses by more than a factor of two because of cluster triaxiality. X-ray mass measurements have much smaller scatter (about a factor of two less than the lensing masses), but they are generally biased toward low values between 5 and $10 \%$. This bias is entirely ascribable to bulk motions in the gas of our simulated clusters. Using the lensing and the X-ray masses as proxies for the true and the hydrostatic equilibrium masses of the simulated clusters and by averaging over the cluster sample, we are able to measure the lack of hydrostatic equilibrium in the systems we have investigated.

Conclusions. Although the comparison between lensing and X-ray masses may be difficult in individual systems due to triaxiality and substructures, using a large number of clusters with both lensing and X-ray observations may lead to important information about their gas physics and allow use of lensing masses to calibrate the X-ray scaling relations.
\end{abstract}

Key words. galaxies: clusters: general - X-ray: galaxies: clusters - gravitational lensing: strong - gravitational lensing: weak

\section{Introduction}

Galaxy clusters are highly important test sites for cosmology. They are the most massive gravitationally bound structures in the universe and, in the framework of the hierarchical structure formation scenario, they are also the youngest systems formed to date. For this reason, the interplay between baryons and dark matter, and its effects on the cluster internal structure, is less important in these than in smaller and older objects. Thus, they are ideal systems for testing the predictions of the cold-dark-matter paradigm on the internal structure of dark-matter halos (Yoshida et al. 2000; Clowe et al. 2004; Markevitch et al. 2004; Sand et al. 2008). Moreover, their mass function is highly sensitive to cosmology, since its evolution traces the growth of the linear density

* Chandra Fellow, Fellow of Michigan Society of Fellows, Department of Physics, University of Michigan, 450 Church St., Ann Arbor, MI 48109-1120, USA. perturbations with exponential magnification (Press \& Schechter 1974; Sheth \& Tormen 2002; Jenkins et al. 2001; Warren et al. 2006).

For these reasons galaxy clusters are being used to constrain the cosmological parameters. Cluster masses are used to measure the time evolution of the mass function, which is compared to the theoretical predictions to constrain the contribution of the components of the universe to the overall density parameter, the equation of state of dark energy, and the normalization of the power spectrum of the initial density fluctuations (e.g. Vikhlinin et al. 2009; Mantz et al. 2009a,b). The measurements can be compared with those obtained from the observation of the universe on larger scales and combined with CMB (see e.g. Komatsu et al. 2009), Supernovae Ia (e.g. Riess et al. 1998, 2004; Perlmutter et al. 1999), and Baryonic-AcousticOscillations observations (e.g. Eisenstein et al. 2005; Percival et al. 2007), in order to put tighter limits to the values of the cosmological parameters. A different approach consists of measuring the concentration-mass relation of clusters and of comparing 
it with the theoretical predictions (e.g. Pointecouteau et al. 2005; Vikhlinin et al. 2005; Buote et al. 2007; Schmidt \& Allen 2007; Comerford \& Natarajan 2007). For example, Buote et al. (2007) find that the concentration-mass relation measured with a sample of 39 clusters agrees closely with the expectations in the framework of the concordance model.

Allen et al. (2008) use the cluster gas fraction to constrain the time evolution of the dark energy component of the universe. The gas fraction measured within a given angular radius is proportional to the distance of the cluster to the power 1.5. Thus, the evolution of the gas fraction can be used to measure the cosmic acceleration (Allen et al. 2004). Combining the results from the $f_{\text {gas }}$ technique with CMB and SNIa data sets, they find that the time evolution of the dark energy equation of state is compatible with the cosmological constant paradigm (see also Ettori et al. 2003).

These techniques rely on scaling relations that link the mass to X-ray observables, such as the temperature, pressure, and luminosity of the X-ray emitting intracluster gas. The scaling relations are predicted to have limited scatter in mass. For example, simulations suggest that the mass- $T_{\mathrm{X}}$ and the mass- $Y_{\mathrm{X}}$ relations just have $15 \%$ and $8 \%$ scatter in mass at fixed $T_{\mathrm{X}}$ and $Y_{\mathrm{X}}$ (e.g. Evrard et al. 1996; Kravtsov et al. 2006). Despite these encouraging predictions, it is clearly essential to measure and calibrate scaling relations empirically. To this purpose, we need to accurately measure the mass profiles of a sample of galaxy clusters that is as large as possible.

There are several methods of deriving the masses of clusters. Two widely used approaches are based on X-ray and lensing observations. X-ray observations allow the cluster mass profiles to be derived by assuming that these systems are spherically symmetric and that the emitting gas is in hydrostatic equilibrium (e.g. Henriksen \& Mushotzky 1986; Sarazin 1988; Ettori et al. 2002). This method has the advantage that, since the X-ray emissivity is proportional to the square of the electron density, it is not very sensitive to projection effects of masses along the line of sight to the clusters. However, it is still not well established how safely the hydrostatic equilibrium approximation can be made.

As the highest mass concentrations in the universe, galaxy clusters are the most efficient gravitational lenses on the sky. Their matter distorts background-galaxy images with an intensity that increases from the outskirts to the inner regions. Strong distortions occur in the cores of some massive galaxy clusters, leading to the formation of "gravitational arcs" and/or to the formation of systems of multiple images of the same source. Weak distortions, which can be only measured statistically, are impressed on the shape of distant galaxies that lie on the sky at large angular distances from the cluster centers (e.g. Bartelmann \& Schneider 2001). Both these lensing regimes can be used to map the mass distribution in galaxy clusters. Determining the masses and the density profiles using lensing offers several advantages over X-ray observations. First, lensing directly probes the cluster total mass, including the dark matter component, without the need to make strong assumptions on the equilibrium state of the lens. Second, mass profiles can be measured over a wide range of scales, from $\lesssim 100 \mathrm{kpc}$ out to the virial radius. The biggest disadvantage is that lensing measures the projected mass instead of the $3 \mathrm{D}$ mass. It is much more sensitive than X-ray methods to projection effects, such as triaxiality and additional concentrations of mass along the line of sight. Given the pros and cons of each method, we can conclude that lensing and X-ray are complementary in many ways. In particular, the comparison of these two mass estimates can greatly help in improving the accuracy of the measurements and understanding the systematic errors.
The picture arising from the comparison of lensing and $\mathrm{X}$-ray mass estimates is puzzling. The two estimates are often discrepant, which implies that we may be missing important ingredients for fully understanding the properties of galaxy clusters. A systematic discrepancy has been revealed in the sense that masses derived from strong lensing are typically higher by a factor 2-3 than masses derived from weak lensing and from the X-ray emitting intra-cluster-medium (ICM) (e.g. Wu \& Mao 1996; Ota et al. 2004; Smith et al. 2005; Riemer-Sørensen et al. 2009). The comparison between weak lensing and X-ray mass estimates is less problematic (see e.g. Ettori \& Lombardi 2003; Allen et al. 2001), but still the lensing masses are on average higher than the X-ray masses by 15-20\% (e.g. Hoekstra 2007; Zhang et al. 2008). Explaining this discrepancy was found to be difficult. It was proposed that X-ray masses could be systematically biased because of mergers or bulk motions of the gas that alter the state of hydrostatic equilibrium (Bartelmann \& Steinmetz 1996; Allen 1998; Mahdavi et al. 2008; Piffaretti \& Valdarnini 2008; Ameglio et al. 2009; Lau et al. 2009). As stated above, lensing masses could also be affected by significant uncertainties (e.g. Bartelmann 1995; Meneghetti et al. 2007).

In this paper, we study the systematic effects in mass measurements done with standard lensing and X-ray techniques. Our work extends some previous work on the systematics on X-ray based mass measurements (see e.g. Rasia et al. 2006; Nagai et al. 2007), which used synthetic X-ray observations of clusters obtained from hydrodynamical simulations to recover their mass distribution under the assumption of hydrostatic equilibrium. Here, we simulate both optical and X-ray observations of the same simulated clusters to be able to compare the mass estimates obtained from both kinds of observations. We use three clusters obtained from hydrodynamical simulations and study them along three independent lines-of-sight.

The paper is organized as follows. In Sect. 2 we describe the sample of numerically simulated galaxy clusters, as well as the techniques used to simulate lensing and X-ray observations. In Sect. 3, we introduce the methods through which the mass estimates are derived from the mock data. In Sect. 4 we show the results of the analyses. Finally, in Sect. 5 we summarize and discuss the major findings of our study.

\section{Simulations}

In this section we describe the numerical methods used in the simulations. We start by considering the $\mathrm{N}$-body/hydrodynamical simulations from which the cluster models are obtained. Then, we explain the lensing and the $\mathrm{X}$-ray simulation pipelines.

\section{1. $N$-body/SPH simulations}

The clusters used in this work are $g 1, g 51$, and $g 72$ from the sample of numerical hydrodynamical simulations presented by Saro et al. (2006). These objects have already been used in several other studies (Dolag et al. 2005; Puchwein et al. 2005; Meneghetti et al. 2007, 2008; Rasia et al. 2006, 2008). Thus, we refer the reader to these papers for more details. They are extracted from a parent simulation of only dark matter (Yoshida et al. 2001) with a box size of $479 h^{-1} \mathrm{Mpc}$ of a flat $\Lambda \mathrm{CDM}$ model with $\Omega_{\mathrm{m}}=0.3$ for the present matter density parameter, $h=0.7$ for the Hubble constant in units of $100 \mathrm{~km} \mathrm{~s}^{-1} \mathrm{Mpc}^{-1}$, $\sigma_{8}=0.9$ for the rms fluctuation within a top-hat sphere of $8 h^{-1} \mathrm{Mpc}$ radius, and $\Omega_{\mathrm{b}}=0.04$ for the baryon density parameter. Each of them was subsequently re-simulated at higher 
mass and spatial resolution using the Zoomed initial condition method (Tormen et al. 1997). The re-simulations were carried out with GADGET- $2^{1}$ (Springel 2005). In the code we include (i) a description of several physical processes in the ICM (see Saro et al. 2006), (ii) a numerical scheme to suppress artificial viscosity far from the shock regions (see Dolag et al. 2005), and (iii) a treatment of chemical enrichment from both SNIa and SNII, as well as from low and intermediate mass stars (Tornatore et al. 2004, 2007). Our simulations assumed the power-law shape for initial stellar mass function, as proposed by Salpeter (1955), and galactic ejects with a speed of $500 \mathrm{~km} \mathrm{~s}^{-1}$. They started with a gravitational softening length fixed at $\epsilon=30 h^{-1} \mathrm{kpc}$ comoving (Plummer-equivalent) and switch to a physical softening length of $\epsilon=5 h^{-1} \mathrm{kpc}$ at $z=5$. The final masses of the $\mathrm{DM}$ and gas particles were set to $m_{\mathrm{DM}}=1.13 \times 10^{9} \mathrm{~h}^{-1} M_{\odot}$ and $m_{\mathrm{GAS}}=1.7 \times 10^{8} h^{-1} M_{\odot}$, respectively.

During the simulation, 92 time slices were saved from redshift 60 to 0 . These are equidistant in time. For the current work, we have used the snapshots corresponding to redshift $z_{1}=0.297$ for $g 1$ and $g 72$ and to redshift $z_{1}=0.2335$ for $g 51$. These redshifts are optimal for both the X-ray and the lensing analyses, since 1) the X-ray surface brightness of these objects is high, and 2) the strength of the lensing signal for sources at $z_{\mathrm{s}} \sim 1$, which is approximately the median redshift of the sources in our simulations, is maximal at $z_{1} \sim 0.3$. All clusters are massive, with masses $M_{200}$ ranging between $\sim 7 \times 10^{14} h^{-1} M_{\odot}$ and $\sim 1.2 \times 10^{15} h^{-1} M_{\odot}$ (see Table 1$) . M_{200}$ is the mass enclosed by the radius $r_{200}$, i.e. the radius within which the average density is 200 times the critical density,

$\rho_{\mathrm{cr}}=\frac{3 H^{2}(z)}{8 \pi G}$

where $H(z)$ is the Hubble parameter.

For each cluster, we select a cube of $20 \mathrm{~h}^{-1} \mathrm{Mpc}$ side length (comoving), centered on the most bound dark matter particle. This is sufficiently large to cover a wide field of view, needed for weak lensing simulations. All the matter contained in this box is projected along three orthogonal lines of sight, in order to produce three lens planes for each cluster. The surface density maps corresponding to the three projections of the clusters $g 1$, $g 51$, and $g 72$ are shown in Fig. 1. The corresponding mass profiles are displayed in Fig. 2. The sample of clusters considered here comprises object with different morphologies, triaxialities, and levels of substructures, although in the X-ray they all appear quite relaxed (see Fig. 4). While the cluster $g 1$ has the most regular morphology, the cluster $g 72$ has a massive companion $\left(\sim 10^{14} h^{-1} M_{\odot}\right)$ located at $\sim 2.5 h^{-1} \mathrm{Mpc}$ from the cluster center. In the projection along the $z$-axis, this secondary clump is much closer to the main halo $\left(\sim 300 h^{-1} \mathrm{kpc}\right)$. Also, the cluster $g 51$ has some substructures within the inner $500 h^{-1} \mathrm{kpc}$, but their masses are lower $\left(\$ 5 \times 10^{12} h^{-1} M_{\odot}\right)$. A massive clump of mass $5 \times 10^{13} h^{-1} M_{\odot}$ orbits at a distance of $3 h^{-1} \mathrm{Mpc}$ from the center.

As demonstrated by the differences between the 2D-mass profiles, all three clusters are triaxial. Their shape is prolate, with the major axis oriented nearly along the $z$-axis of the simulation box in the cases of $g 51$ and $g 72$, and nearly along the $x$-axis in the case of $g 1$. The axis ratios, measured at $r_{200}$ by calculating the cluster inertia ellipsoids, are listed in Table 1, together with some other relevant properties. We also report there the angles between the main axes of the clusters and the $x$-, $y$-, and $z$-axes of the simulation boxes.

${ }^{1}$ http://www.MPA-Garching. MPG.DE/gadget/
The clusters are described well by Navarro-Frenk-White (NFW) density profiles (Navarro et al. 1997), whose functional form is given by

$\rho_{\mathrm{NFW}}(r)=\frac{\rho_{\mathrm{s}}}{r / r_{\mathrm{s}}\left(1+r / r_{\mathrm{s}}\right)^{2}}$

where $\rho_{\mathrm{s}}$ and $r_{\mathrm{s}}$ are the characteristic density and the scale radius, respectively. The characteristic density is often written in terms of the concentration parameter, $c_{200}=r_{200} / r_{\mathrm{s}}$, as

$$
\rho_{\mathrm{s}}=\frac{200}{3} \rho_{\mathrm{cr}} \frac{c_{200}^{3}}{\left[\ln \left(1+c_{200}\right)-c_{200} /\left(1+c_{200}\right)\right]}
$$

The last two columns of Table 1 report the best-fit concentrations and scale-radii obtained by fitting the 3D dark-matter density profiles of the clusters with the formula in Eq. (2). The radial fits are done between $10 h^{-1} \mathrm{kpc}$ and $r_{200}$.

In the remainder of the paper, we refer to the projections of cluster $g \mathrm{~N}$ along the simulation axis $\mathrm{X}$ using the abbreviation $g \mathrm{~N}-\mathrm{X}$.

\subsection{Lensing simulations}

In this section we describe the ray-tracing algorithms used to derive the lensing distortion fields of our simulated clusters. For a summary of the lensing definitions used through the rest of the paper, we refer the reader to Appendix A. In the following, we make use of the standard thin-screen approximation; i.e., we assume that the deflections occur on a plane perpendicular to the line-of-sight, passing through the cluster center. This is justified since the distances between the observer and the lens and between the lens and the background sources are much larger than the sizes of the clusters.

The particles projected on each lens plane are used to calculate the deflection angles of bundles of light rays. The light rays are traced from the observer position towards the background sources through two regular grids with different spatial resolutions. The inner $1.5 \times 1.5 h^{-2} \mathrm{Mpc}^{2}$ region around the cluster center is sampled with $2048 \times 2048$ light rays. This guarantees sufficient spatial resolution for reproducing accurately the positions of multiple images in the strong lensing (SL) regime (Meneghetti et al. 2007). For the weak lensing (WL) regime, we need to sample a much wider area, while the spatial resolution is less important. Thus, we cover the whole lens plane with a grid of $4096 \times 4096$ light rays. The deflection angles are computed using a tree-based code, which works as follows. First, it ranks the particles based on their distances from the light ray positions, building a Barnes \& Hut oct-tree in 2D. The contributions to the deflection angles from nearby and distant particles are calculated separately using direct summation or higher-order Taylor expansions of the deflection potential around the light-ray positions. Precisely, given a light ray at position $\boldsymbol{R}$ in physical units, which corresponds to an angular position $\boldsymbol{\theta}=\boldsymbol{R} / D_{1}$, the contribution to its deflection angle by a system of mass elements, $m_{a}$ at positions $\boldsymbol{R}_{a}(a=1,2, \ldots, N-1, N)$, with center of mass $\boldsymbol{R}_{\mathrm{CM}}$, and with $\left|\boldsymbol{R}-\boldsymbol{R}_{\mathrm{CM}}\right| \ll\left|\boldsymbol{R}_{a}-\boldsymbol{R}_{\mathrm{CM}}\right|$ for all the mass elements $a$, is

$$
\begin{aligned}
\alpha_{i}(\boldsymbol{R})= & \frac{4 G M}{c^{2}}\left[F_{1}\left(R^{\prime}\right) \delta^{i j}+F_{2}\left(R^{\prime}\right) Q^{i j}+\frac{1}{2} F_{3}\left(R^{\prime}\right)\left(R^{\prime k} Q_{k n} R^{\prime n}\right) \delta^{i, j}\right. \\
& \left.+\frac{1}{2} F_{4}\left(R^{\prime}\right) P^{i j}\right] R_{j}^{\prime}
\end{aligned}
$$



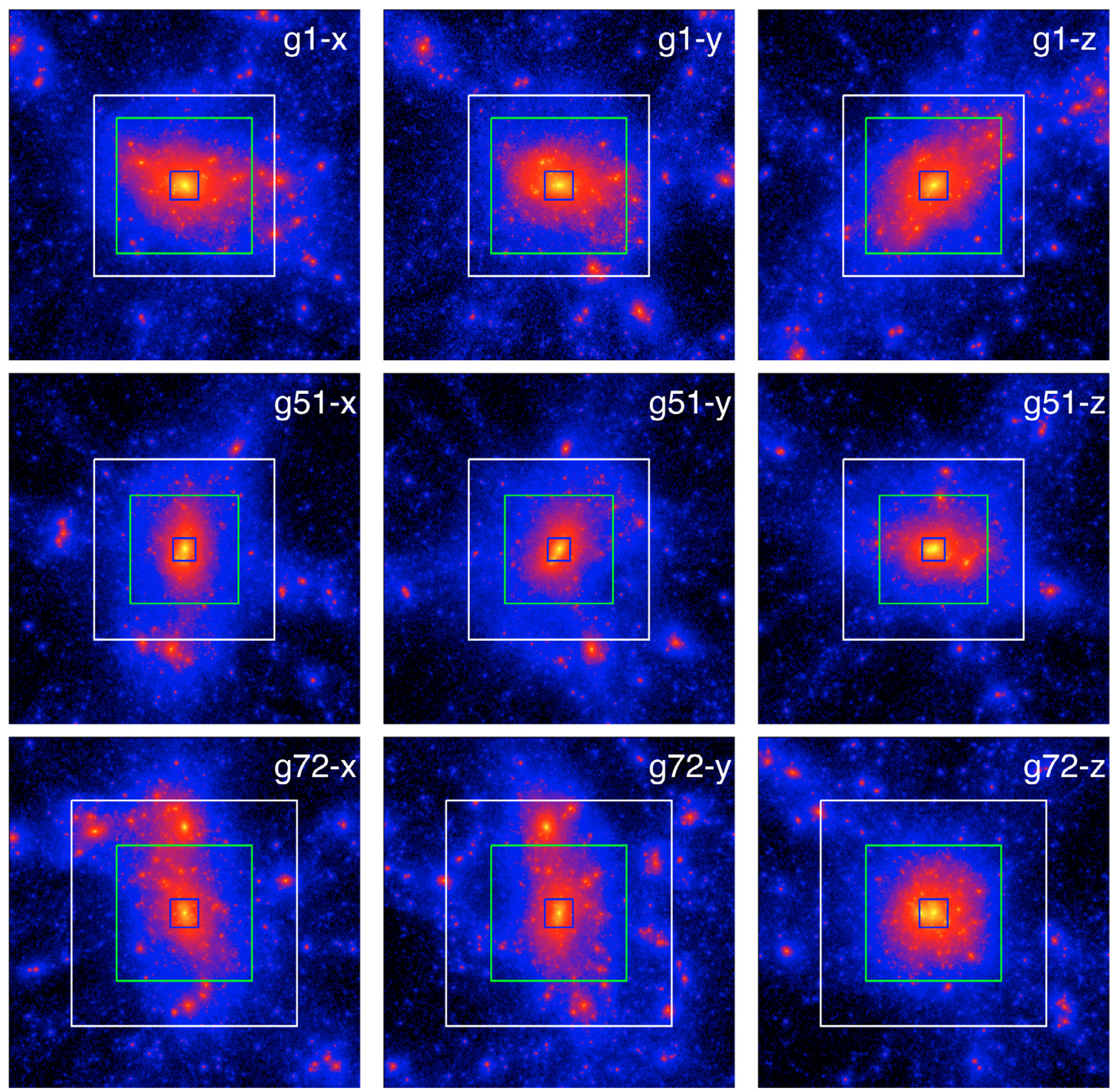

Fig. 1. Surface density maps of the three projections of the clusters $g 1$ (top panels), g51 (middle panels), and $g 72$ (bottom panels) used in this work. The size of each map is $10 h^{-1} \mathrm{Mpc}$ comoving. Such a scale corresponds to $\sim 51.85$ arcmin for $g 51$ and to $\sim 41.47$ arcmin for $g 1$ and $g 72$. In each panel, the inner, middle, and outer boxes indicate the fields-of-view used for the HST, Chandra, and SUBARU simulated observations, respectively (see text for more details).

where $M$ is the total mass of the system, $\boldsymbol{R}^{\prime}=\boldsymbol{R}-\boldsymbol{R}_{\mathrm{CM}}, \delta^{i j}$ is the Kroneker function, and the tensors $P$ and $Q$ are defined as

$Q_{i j}=\frac{1}{M} \sum_{a=1}^{a=N} m_{a} R_{i}^{\prime a} R_{j}^{\prime a}$,
$P_{i j}=\frac{1}{M} \sum_{a=1}^{a=N} m_{a}\left|R^{\prime a}\right|^{2} \delta_{i j}$.

Assuming a Plummer softening to avoid the deflection angles diverging, the $F_{k}\left(R^{\prime}\right)$ functions are defined as

$$
\begin{gathered}
F_{1}\left(R^{\prime}\right)=\frac{1}{\left(R^{\prime 2}+s^{2}\right)} \\
F_{2}\left(R^{\prime}\right)=\frac{-2}{\left(R^{\prime 2}+s^{2}\right)^{2}}
\end{gathered}
$$

$F_{3}\left(R^{\prime}\right)=\frac{8}{\left(R^{\prime 2}+s^{2}\right)^{3}}$

$F_{4}\left(R^{\prime}\right)=\frac{-2}{\left(R^{\prime 2}+s^{2}\right)^{2}}$.

Nearby particles are treated as point lenses and Eq. (4) reduces to

$\alpha_{i}(\boldsymbol{R})=\frac{4 G M}{c^{2}} R_{i}^{\prime} F_{1}\left(R^{\prime}\right)$.

The fraction of particles that are evaluated with Eqs. (4) or (11) is set by the Barnes-Hut opening criterion, $\theta_{\mathrm{BH}}$ (see e.g. Springel 2005), which we fix at $\theta_{\mathrm{BH}}=0.4$. As shown by Aubert et al. (2007), the optimal softening length $s$ depends on the resolution of the simulation. We performed several tests to determine which values to use. Doing ray-tracing through NFW halos sampled with a similar number of particles as our simulated clusters, 
M. Meneghetti et al.: Weighing simulated galaxy clusters using lensing and X-ray

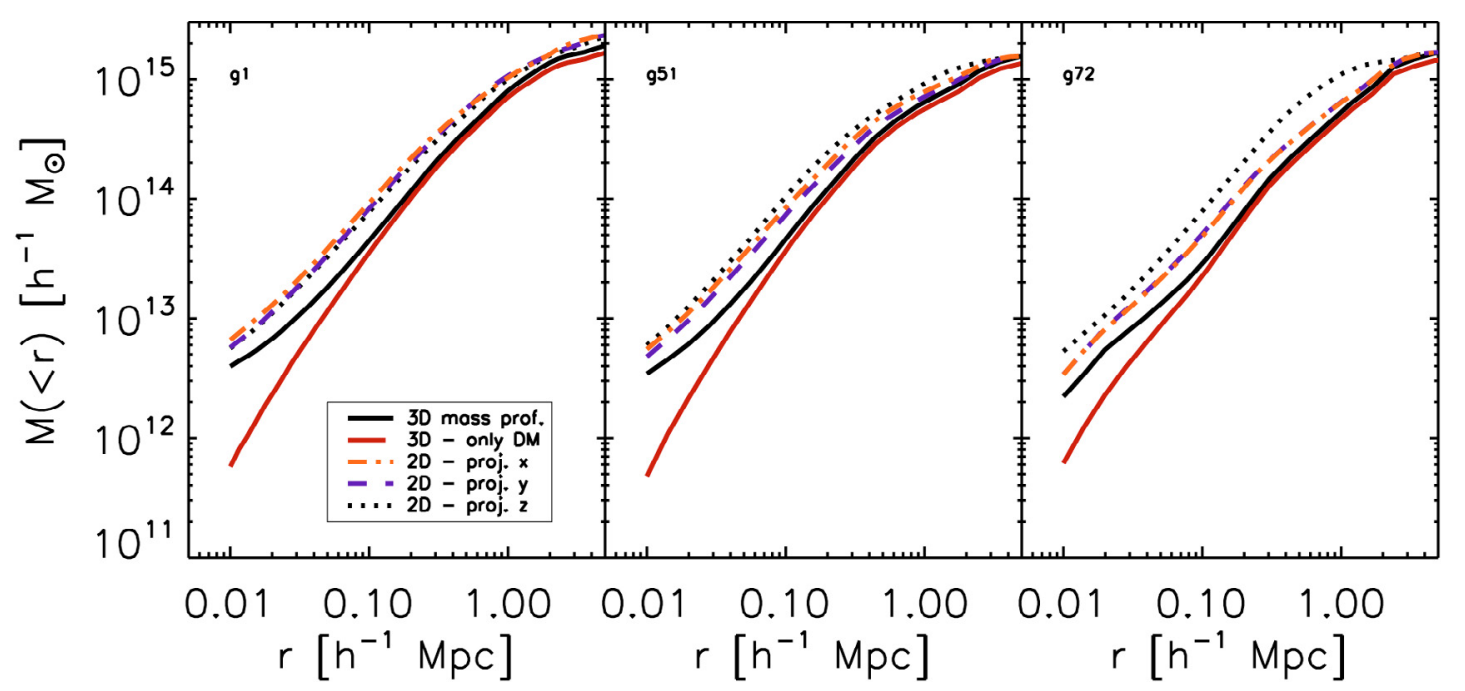

Fig. 2. Mass profiles of the clusters $g 1, g 51$, and $g 72$. The solid black and red lines indicate the total and DM only 3D-mass profiles, respectively. The total 2D-mass profiles corresponding to the $x, y$, and $z$ projections of each cluster are given by the dotted, dashed, and dash-dotted lines.

Table 1. Main properties of the simulated clusters used in this work.

\begin{tabular}{lcccccccccc}
\hline \hline Cluster & Redshift & $\begin{array}{c}r_{200} \\
{\left[h^{-1} \mathrm{Mpc}\right]}\end{array}$ & $\begin{array}{c}M_{200} \\
{\left[h^{-1} M_{\odot}\right]}\end{array}$ & $b / a$ & $c / a$ & $\begin{array}{c}\theta_{x} \\
{[\mathrm{deg}]}\end{array}$ & $\begin{array}{c}\theta_{y} \\
{[\mathrm{deg}]}\end{array}$ & $\begin{array}{c}\theta_{z} \\
{[\mathrm{deg}]}\end{array}$ & $c_{200}$ & $\begin{array}{c}r_{\mathrm{s}} \\
{\left[h^{-1} \mathrm{Mpc}\right]}\end{array}$ \\
\hline g1 & 0.297 & 1.54 & $1.14 \times 10^{15}$ & 0.64 & 0.57 & 33.3 & 57.4 & 96.1 & 4.62 & 0.310 \\
g51 & 0.2335 & 1.39 & $7.85 \times 10^{14}$ & 0.78 & 0.65 & 81.5 & 75.59 & 16.8 & 5.37 & 0.241 \\
g72 & 0.297 & 1.30 & $6.83 \times 10^{14}$ & 0.31 & 0.29 & 98.9 & 92.8 & 9.4 & 3.99 & 0.299 \\
\hline
\end{tabular}

we verified that a softening scale of $5 h^{-1} \mathrm{kpc}$ is appropriate for reliably reproducing the deflection angle field of the input models over the range of scales relevant for both strong and weak lensing.

Having obtained the deflection angle maps, we apply the cluster distortion fields to the images of a large number of background galaxies. While doing so, we simulated optical observations of each cluster under the different projections. For this purpose, we used the code described in Meneghetti et al. (2008) (quoted as SkyLens hereafter), which has been recently further developed. In short, the code uses a set of real galaxies decomposed into shapelets (Refregier 2003) to model the source morphologies on a synthetic sky. In the current version of the simulator, the shapelet database contains $\sim 3000$ galaxies in the $z$-band from the GOODS/ACS archive (Giavalisco et al. 2004) and $\sim 10000$ galaxies in the $B, V, i, z$ bands from the HubbleUltra-Deep-Field (HUDF) archive (Beckwith et al. 2006). Most galaxies have spectral classifications and photometric redshifts available (Benítez 2000; Coe et al. 2006), which are used to generate a population of sources whose luminosity and redshift distributions resemble those of the HUDF.

SkyLens allows us to mimic observations with a variety of telescopes, both from space and from the ground. We simulate wide field observations, on which we carry out a weak lensing analysis, using the SUBARU Suprime-Cam. We simulate Hubble-Space-Telescope observations of the cluster central regions using the Advanced Camera for Surveys (ACS). All simulations include realistic background and instrumental noise. As an example, in Fig. 3 we show two color-composite images of one simulated galaxy cluster (although in the rest of the paper, we use simulated observation in a single band). The left and the right panels show the results of simulated observations with SUBARU and with HST, respectively. The galaxy colors are realistically reproduced by adopting 22 SEDs to model the background galaxies, following the spectral classifications published by Coe et al. (2006).

In the HST image, several blueish arcs and arclets are visible behind the cluster galaxies. These are originated by background spiral and irregular galaxies strongly lensed by the foreground cluster.

In our analysis, we used simulated observations with the following characteristics. For the SUBARU simulations, we assumed an exposure time of $6000 \mathrm{~s}$ in the $I$ band, with a seeing of $0.6^{\prime \prime}$. The PSF is assumed to be isotropic and modeled using a 2D Gaussian. For the HST simulations, we assumed an exposure time of $7500 \mathrm{~s}$ with the $F 775 \mathrm{~W}$ filter. The fields-of-view adopted for the HST and SUBARU simulations were overlaid to the surface density maps in Fig. 1 (blue inner- and white outerboxes, respectively). These fields of view do not correspond to the true fields-of-view of the ACS and Suprim-CAM mounted on the HST and on the SUBARU telescope. For computational efficiency we limit the fields-of -view in the HST simulated observations to 120 arcsec, which is wide enough to contain the Einstein rings of our clusters. For the SUBARU simulations, the fields-of-view are defined to correspond to the same comoving scale on the lens plane, i.e. $4 h^{-1} \mathrm{Mpc}$, for clusters $g 1$ and $g 51$. For cluster $g 72$, we simulate a wider field-of-view, corresponding to $5 \mathrm{~h}^{-1} \mathrm{Mpc}$ comoving in order to include a large substructure in the observation.

\subsection{X-ray simulations}

The X-ray images of our simulated clusters are produced with the code X-ray MAp Simulator (XMAS). The software is presented in Gardini et al. (2004) and Rasia et al. (2008), where a full description of the simulation pipeline can be found. It generates synthetic event files that have the same format as real 

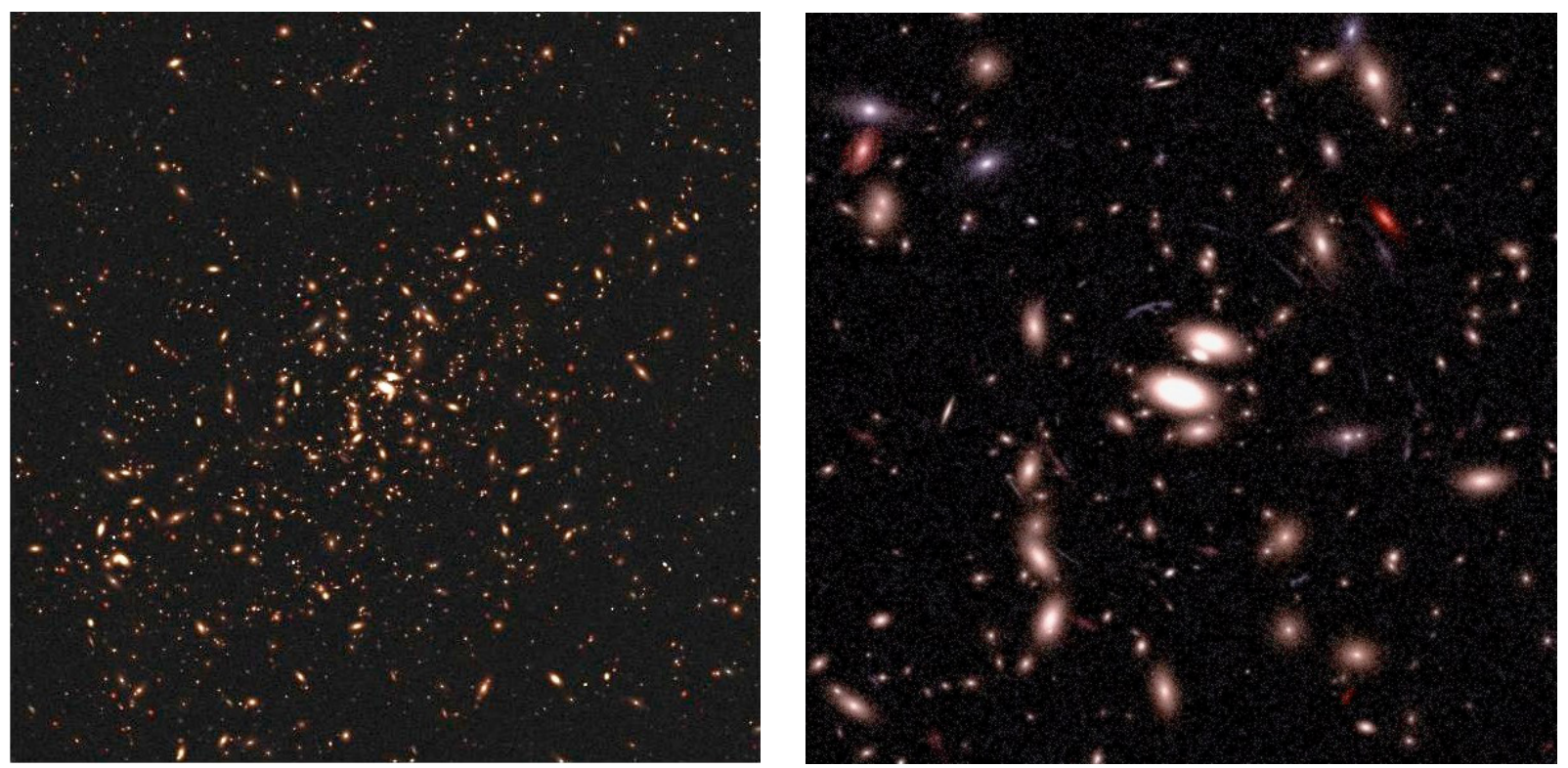

Fig. 3. Left panel: color-composite image of a simulated galaxy cluster, obtained by combining three SUBARU exposures of $2500 \mathrm{~s}$ each in the $B, V, I$ bands. The field-of-view corresponds to $\sim 450^{\prime \prime} \times 450^{\prime \prime}$. Right panel: an HST/ACS composite image of the the central $100^{\prime \prime} \times 100^{\prime \prime}$ of the same cluster. The image has been produced by combining mock observations with the filters $F 475 \mathrm{~W}, F 555 \mathrm{~W}$ and $F 775 \mathrm{~W}$.

observations. In this work, which is not intended to exploit any $\mathrm{X}$-ray calibration issue, we assumed a constant response over the detector. In particular, the response matrix files and ancilliary response file were those of the aimpoint of ACIS-S3 CCD on board of Chandra telescope. For each cluster projection we produced an X-ray image. The X-ray images have a field of view of 16 arcmin on a side, which corresponds to $2.5 h^{-1} \mathrm{Mpc}$ at $z=0.2335$ and to $2.97 h^{-1} \mathrm{Mpc}$ at $z=0.297$ for the considered cosmology. The Chandra field-of-view is overlaid on the surface density maps of the nine clusters in Fig. 1 (green box). The background is not included a priori since Rasia et al. (2006) shows that it does not induce any systematics on the bias. The spectral model used to generate the photons considers the contributions from the different metal species present in the simulation: $\mathrm{C}, \mathrm{N}$, $\mathrm{O}, \mathrm{Mg}, \mathrm{Si}$, and $\mathrm{Fe}$. The exposure time is $500 \mathrm{ks}$. The images for all the cluster projections analyzed in this paper are shown in Fig. 4. The color bar on the bottom allows to convert the color levels into counts per pixel.

\section{Analyses}

In this section, we describe the methods used for analyzing the previously outlined simulations. Firstly, we consider the mass estimates based on strong and weak lensing separately. Then we also discuss a nonparametric method that combines both the lensing regimes. Finally, we consider two methods for deriving the cluster masses from the X-ray simulated data.

\subsection{Strong lensing}

The strong lensing analysis is performed by using the public software Lenstool (Kneib et al. 1993). This is very welldeveloped tool for strong lensing parametric reconstructions, which allows fitting the observed strong lensing features in a cluster field through the combination of several mass components, each of which can be characterized by a density profile and by a projected shape (ellipticity and orientation). The code uses a Bayesian approach to finding the best-fit lens model and to estimating the errors on the free parameters. We refer the reader to the paper by Jullo et al. (2007) for a more detailed description of the many options available in this software.

Lenstool allows the user to choose among several available density profiles to describe the lens components. In this work, we use their implementation of the NFW profile (Golse et al. 2002) to model the main cluster halo. The functional form of this profile is given in Eq. (2). Moreover, we add several subcomponents, representing the contribution from the most massive galaxies in the cluster. As shown in some previous studies, it is important to include the cluster members in the model, because they can affect the positions and the magnifications of the strong lensing features (Meneghetti et al. 2003a; Meneghetti et al. 2007). These are modeled using pseudo-isothermal-ellipticalmass-distributions (PIEMD) described by the following density profile:

$\rho_{\text {PIEMD }}(r)=\frac{\rho_{0}}{\left(1+r^{2} / r_{\text {core }}^{2}\right)\left(1+r^{2} / r_{\text {cut }}^{2}\right)}$.

We chose the PIEMD model because this is widely used for modeling the lensing properties of cluster galaxies in observations (see e.g. Limousin et al. 2007; Riemer-Sørensen et al. 2009; Donnarumma et al. 2009, for some recent references). As shown in the previous equation, this profile is parametrized by a central density $\rho_{0}$, which is linked to the central velocity dispersion $\sigma_{0}$, and by two characteristic radii, namely the core radius $r_{\text {core }}$ and the cut-off radius $r_{\text {cut }}$. To incorporate the galaxy population into the global lens model, we used the same approach used by Limousin et al. (2007), who scale the parameters as a function of the luminosity (or equivalently of the mass). The scaling relations are given by

$$
\begin{aligned}
r_{\text {core }} & =r_{\text {core }}^{\star}\left(\frac{L}{L^{\star}}\right)^{1 / 2} \\
r_{\text {cut }} & =r_{\text {cut }}^{\star}\left(\frac{L}{L^{\star}}\right)^{1 / 2} \\
\sigma_{0} & =\sigma_{0}^{\star}\left(\frac{L}{L^{\star}}\right)^{1 / 4}
\end{aligned}
$$


M. Meneghetti et al.: Weighing simulated galaxy clusters using lensing and X-ray
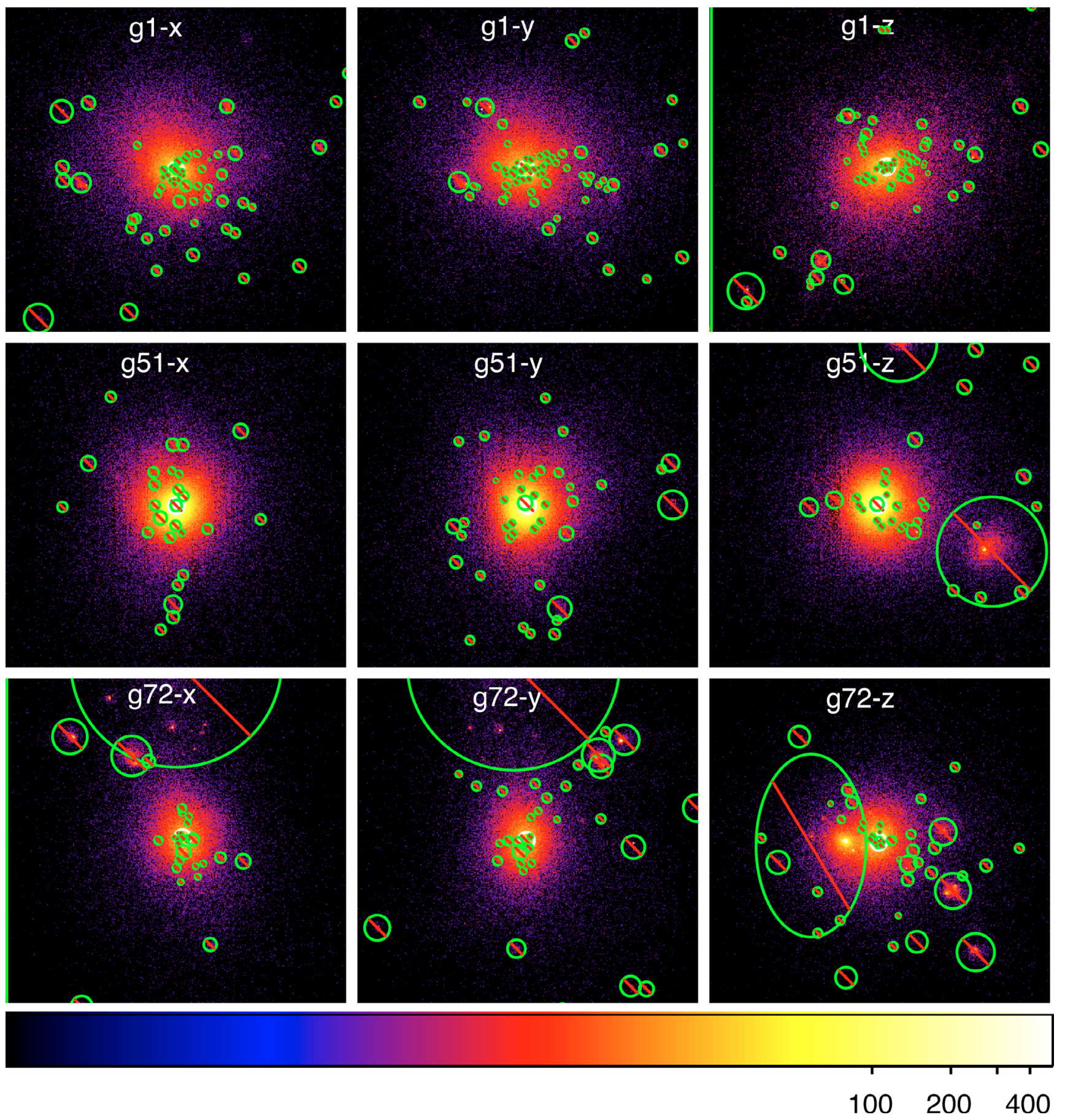

Fig. 4. X-ray maps of the three projections of the clusters $g 1$ (top panels), g51 (middle panels), and $g 72$ (bottom panels) used in this work. The size of each map is 16 arcmin, which corresponds to $2.5 h^{-1} \mathrm{Mpc}$ for $g 51$ and to $2.97 h^{-1} \mathrm{Mpc}$ for $g 1$ and $g 72$. In each panel, we display the masked regions. The dense cold blobs are encircled in green, while we indicate the excluded central region in white. The color bar on the bottom allows to convert colors into counts.

where $L^{\star}$ is a reference luminosity and the quantities $r_{\text {core }}^{\star}, r_{\text {cut }}^{\star}$, and $\sigma_{0}^{\star}$ are the corresponding core and cut radii and central velocity dispersion, respectively. In our analysis, we assumed a constant mass-to-light ratio for all the cluster members. Such an assumption is generally adopted when studying real clusters.

Observationally, the galaxies to be included in the model should be selected as those lying in the cluster red sequence and being brighter than a given apparent luminosity (e.g. Limousin et al. 2007). Of course what matters for lensing is not the luminosity but the mass, which is assumed to be traced by the light. Indeed, the minimal luminosity should be interpreted as a minimal mass. Working with simulations, we identify the cluster galaxies using the SUBFIND code (Springel et al. 2001) and then apply a selection based directly on the stellar mass. SUBF IND decomposes the cluster halo into a set of disjoint substructures and then identifies each of them as a locally overdense region in the density field of the background halo. In our reconstructions, we include those galaxies that have stellar mass $M_{\text {stars }} \geq 10^{9} h^{-1} M_{\odot}$ and that are contained in a region of $500 \mathrm{~h}^{-1} \mathrm{kpc}$ around the cluster center. This is typically more than three times the size of the Einstein rings of the clusters in our sample. The orientation and the ellipticity of each galaxy are measured from the 

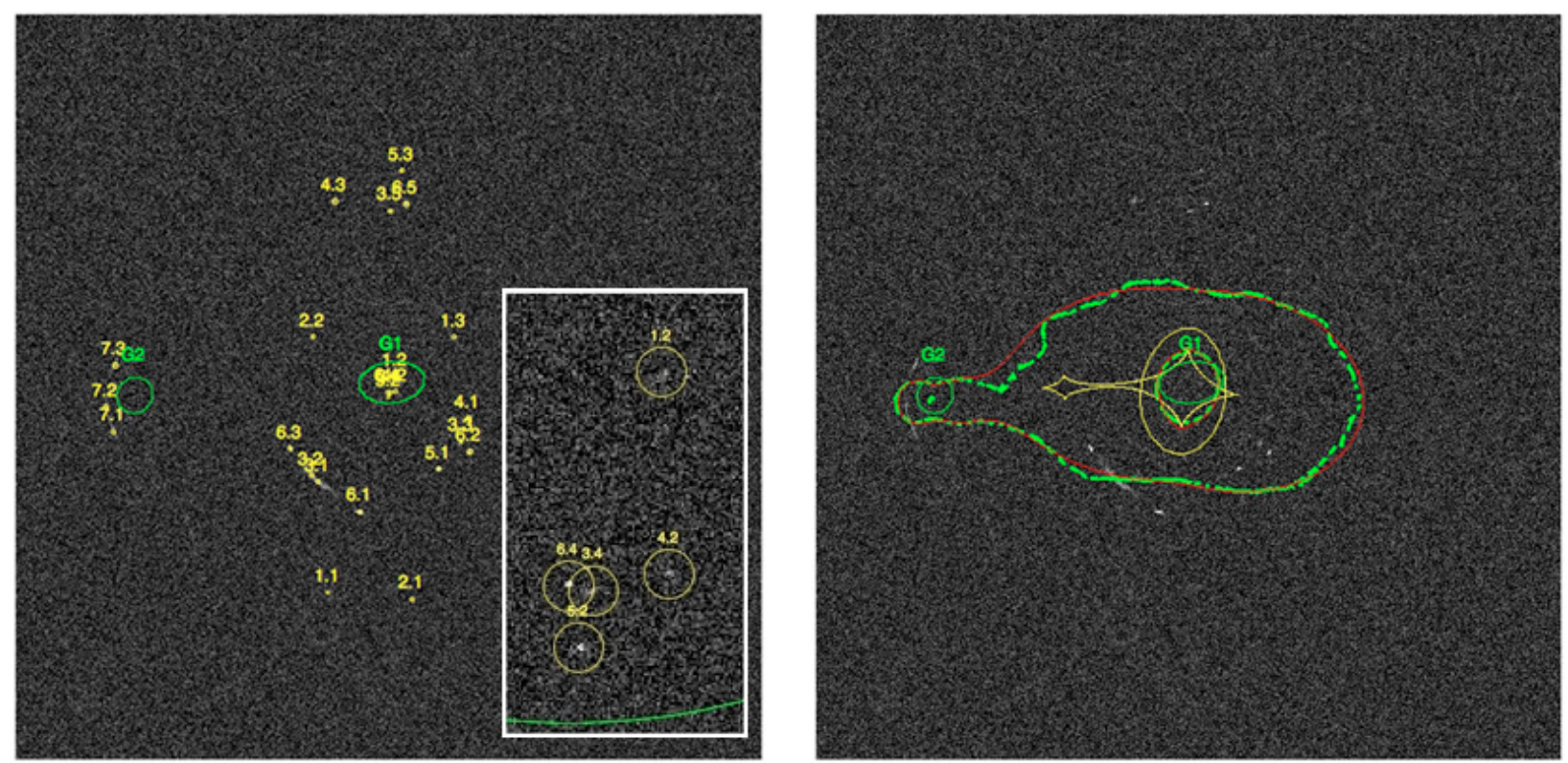

Fig. 5. Left panel: HST-like view over the central $120^{\prime \prime} \times 120^{\prime \prime}$ of cluster $g 1$ (projected along the $y$ axis). The light emission from the cluster galaxies has been removed to avoid confusion with the background multiple images. The identified systems are shown in yellow. The images $i$ belonging to the source $n$ are indicated with the labels $n . i$. The inset on the left shows a zoom over the very central region of the image, where the central images 1.2, 3.4, 4.2, 5.2, and 6.4 are located. We also show in green the location of the cluster members that have been included in the lens reconstruction. Right panel: the result of the reconstruction obtained by using Lenstool with the lensing constraints shown in the left panel. The critical lines for sources at redshift 1.7 are shown in red and the corresponding caustics in yellow. We also overplot the true critical lines of the cluster in green.

distribution of the star particles belonging to it. Following this procedure, we typically end up with catalogs of several tenth of cluster members.

The Brightest-Central-Galaxy (BCG) is included in the lens model by optimizing its parameters individually, rather than scaling them with the luminosity/mass. Since the BCG forms in the simulations in a strong cooling region, we assumed it might have significantly different properties compared to the other cluster members. Thus, we prefer to treat it individually. Analogously, we use individual optimization with some other cluster members which lay particularly close to some multiple image systems. Indeed, their influence on the local lensing properties of the cluster requires to be carefully modeled.

The total number of free parameters in the model depends on the complexity of the lens. Usually we consider a clusterscale mass component, a galaxy-scale component to describe the $\mathrm{BCG}$, and other galaxy-scale terms to incorporate the relevant cluster members.

We distribute the sources behind the clusters so to have 2-7 strong lensing systems available for the optimization. For this condition to be satisfied, we randomly distribute few sources in a shell surrounding the lens caustics, enhancing the chances that they are strongly lensed. Then, we visually check whether the multiple images belonging to each source are detectable in the simulation and then retain those systems that are useful for the strong lensing analysis. The optimization is done using the Bayesian method implemented in LENSTOOL with optimization rate $\delta \lambda=0.1$. We assume the uncertainty in the lensed image positions to be $\sigma_{\mathrm{I}}=0.3^{\prime \prime}$.

In Fig. 5, we illustrate the reconstruction of cluster $g 1-y$. The system has a massive galaxy at the center, labeled G1, and another massive galaxy located $\sim 45^{\prime \prime}$ west of the BCG (G2). Thus, we fit the lensing observables using three lens components, namely the main halo, the BCG, and a secondary PIEMD clump coincident with the other massive galaxy. In this particular case, adding additional cluster members does not affect the reconstruction. Among the sources, which were distributed along the caustics of the input cluster lens, seven of them produce multiple-image sytems detectable in this deep exposure (7500 s) in the $F 775 W$ filter. More precisely, two sources (source 3 and 6) produce five images, while the other sources are imaged into triplets. These are displayed in the left panel of Fig. 5. The bright knots in the multiple images of the same source are marked with points and identified with labels. The first digit corresponds to the source number, while the last indicates the multiplicity of the image to which the knots belong. For the sake of simplicity, the simulation is shown without including the light emission from the cluster members neither from other background sources that are not strongly lensed. The central image of source 2 cannot be detected by eye, so it is not used in the reconstruction. The other central images of sources $1,3,4,5,6$ lie behind the BCG but are detectable in the BCG subtracted frame. Using these observables, the reconstruction converges, finding a good fit to the lensing features ( $\chi^{2}=18$ for 21 degrees of freedom). The best-fit model consists of an NFW halo with concentration $c=10.57_{-1.81}^{+2.82}$ and scale radius $r_{\mathrm{s}}=29.07_{-1.85}^{+20.68}$ arcsec (corresponding to $90_{-6}^{+64} h^{-1} \mathrm{kpc}$ ). The galaxies $\mathrm{G} 1$ and $\mathrm{G} 2$ have velocity dispersions of $340_{-30}^{+21} \mathrm{~km} \mathrm{~s}^{-1}$ and $269_{-17}^{+12} \mathrm{~km} \mathrm{~s}^{-1}$, respectively. To illustrate the result of the modeling, we show in the right panel of Fig. 5 the true and the reconstructed critical lines of the lens, assuming a source redshift of $z_{\mathrm{s}}=1.72$ (source 3 ). Both the tangential and the radial critical lines of the cluster are generally reproduced quite well by the model. The largest differences are on those portions of the critical lines along which fewer lensing constraints are present.

The inner projected mass profile derived from the model, $M(<R)$, is shown in Fig. 6, where we also show the true profile of the cluster acting as lens in this simulation. Since the model 


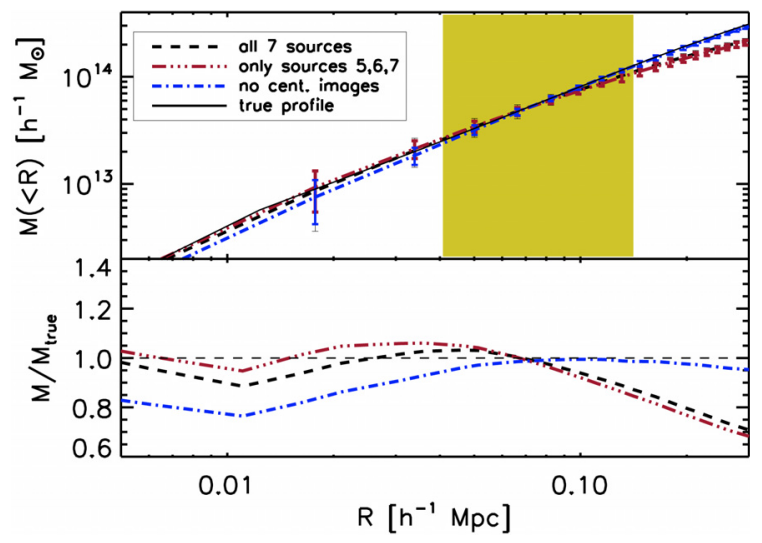

Fig. 6. Results of the strong lensing analysis. The total projected mass profile of the inner region of cluster $g 1-y$ as recovered from the strong lensing mass reconstruction using LENSTOOL. The dashed line shows the result obtained by using seven multiple-image systems. The red three-dot-dashed line shows the mass profile if only three multipleimage systems are used. The blue dot-dashed line indicates the mass profile recovered by fitting all the 7 multiple-image systems, but assuming that all the central images are not detectable. The lensing constraints here are shown in the left panel of Fig. 5. Finally, the true mass profile, as drawn from the particle distribution in the input cluster, is given by the solid line. The shaded region shows the radial range of the tangential strong lensing constraints. The bottom panel shows the ratios between the recovered mass profiles and the true mass profile.

reproduces the lens tangential critical line well, it is not surprising that the model is very reliable at estimating the mass enclosed in the strong lensing region. The yellow area in the figure indicates the radial range of the multiple images, excluding the central images, which are located at $R \lesssim 10 h^{-1} \mathrm{kpc}$. The reconstruction reproduces the true mass profile well up to $\sim 150 h^{-1} \mathrm{kpc}$ from the center, where the deviation from the true mass profile is $\lesssim 10 \%$. At larger radii, the differences become significant. Thus, extrapolating the strong lensing model to distances where no strong lensing features are observed may result in very incorrect mass estimates. This issue is discussed in more detail in Sect. 4.

To evaluate how the reliability of the model degrades by reducing the number of constraints, we performed another reconstruction using only one system with five images (arising from the source 6) and two triplets (source 5 and 7). The final reconstruction did not differ significantly from the previous one. The projected mass profile for this new lens model is given in Fig. 6. This result shows that reliable reconstructions can be achieved even with a limited number of lensing constraints, if they are optimally distributed across the cluster. We also attempted a reconstruction by neglecting the central images (and using all the seven lensed systems). This is likely to be a realistic situation, since the central images are generally demagnified and hidden behind the BCG, hence difficult to detect. In this case, the mass enclosed by the strong lensing region is again correctly estimated, but the reconstructed profile deviates more from the true one at small radii, as shown in Fig. 6. In the following, the SL models are constructed using also the central images, when they are detectable in the galaxy subtracted frames.

\subsection{Weak lensing}

The weak lensing measurements are done using the standard KSB method, proposed by Kaiser et al. (1995) and subsequently extended by Luppino \& Kaiser (1997) and by Hoekstra et al. (1998). This method is now internally implemented in Skylens.
The galaxy ellipticities are measured from the quadrupole moments of their surface brightness distributions, corrected for the PSF, and used to estimate the reduced shear under the assumption that the expectation value of the intrinsic source ellipticity vanishes (see Eq. (A.14)).

By selecting the galaxies with $S / N>10$, we end up with catalogs of galaxy ellipticities containing $\sim 30$ sources/sq. arcmin. The median redshift of these sources is $z_{\mathrm{s}, \text { true }} \sim 1.05$. In the following analysis we assume that all sources have the same redshift of $z_{\mathrm{s}}=1$. Furthermore, we assume that we can separate the population of background galaxies perfectly from the foreground cluster members. This is intentionally very optimistic, since we aim here at verifying the capabilities of several lensing methods to retrieve the cluster mass in the best possible conditions. The misidentification of cluster members as background galaxies leads to a dilution of the lensing signal, which leads to erroneous mass estimates (see e.g. Medezinski et al. 2007). We will address in more detail this issue in a forthcoming paper. When increasing the distance from the cluster center, the probability that nearby substructures or additional mass clumps affect the mass estimates becomes higher. In this work, we have not included the effects of uncorrelated large-scale-structures (LSS) on the weak-lensing signal. The effects of the LSS on the weak lensing mass estimates have been discussed in detail in several other papers (Cen 1997; Metzler et al. 1999; Hoekstra 2001, 2003; White \& Vale 2004). Uncorrelated LSS introduce a noise in the mass estimates, but the importance of matter along the line sight is fairly small for rich clusters at intermediate redshifts, like those in our sample, provided that the bulk of the sources are at high redshift compared to the cluster. Given that we are considering all the mass in cylinders of height $20 \mathrm{~h}^{-1} \mathrm{Mpc}$ in the lensing simulations, the effects of the correlated large-scale structure is partially included. Clowe et al. (2004) have studied the weak lensing signal of our same clusters (but in a pure dark matter version) in their cosmological environment. They find that including all the matter in a cylinder of height $\sim 100 h^{-1} \mathrm{Mpc}$ around the clusters only results in small scatter being added to the measured cluster masses, compared to simulations where a cylinder of only $\sim 10 h^{-1} \mathrm{Mpc}$ was used. These results agree with the findings of Reblinsky \& Bartelmann (1999).

The cluster masses are derived with the following approaches:

NFW fit of the tangential shear profile: assuming that the cluster is described well by an NFW density profile, we use the corresponding formula for the reduced shear to fit the azimutally averaged profile of the tangential component of the reduced shear. For the NFW profile, the formulas for the radial profiles of the shear and of the convergence can be found in Bartelmann (1996) and in Meneghetti et al. (2003b). The tangential component of the reduced shear is given by

$g_{+}=-\operatorname{Re}\left[g \mathrm{e}^{-2 \mathrm{i} \phi}\right]$,

where the angle $\phi$ specifies the direction from the galaxy centroid towards the center of the cluster, which we identify with the position of the BCG. The cross component of the reduced shear is given by

$g_{\times}=-\operatorname{Im}\left[g \mathrm{e}^{-2 \mathrm{i} \phi}\right]$.

If the distortion is caused by lensing, this component of the shear should be zero.

In Fig. 7, we show the radial profiles of both the components of the shear for the cluster $g 1-y$, measured out to large 


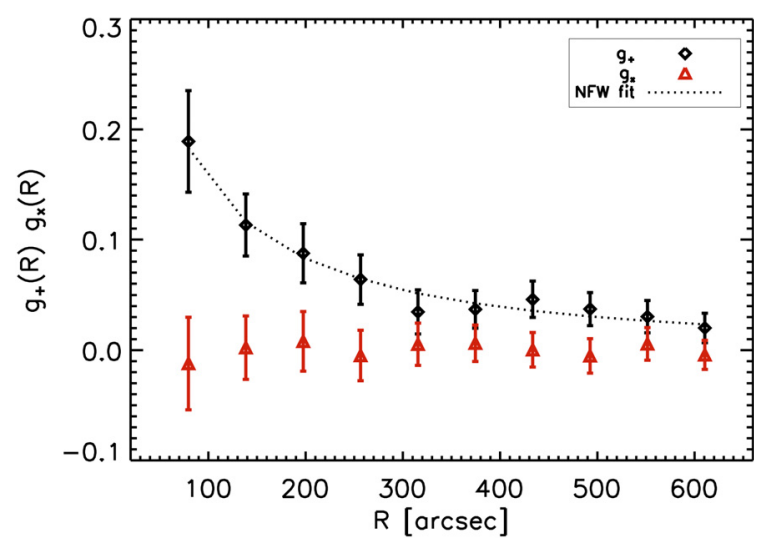

Fig. 7. Radial profiles of the tangential and of the cross components of the reduced shear measured from the center of cluster $g 1-y$. The dotted line shows the best-fit NFW model.

radii ( $\sim 3 h^{-1} \mathrm{Mpc}$ from the cluster center). The tangential component is well-fitted by an NFW profile with $c=4.82 \pm 0.64$ and $r_{\mathrm{s}}=0.307 \pm 0.048 h^{-1} \mathrm{Mpc}$. As expected in the absence of systematics, the cross component of the shear is consistent with zero.

Aperture mass densitometry: the aperture mass densitometry (Fahlman et al. 1994; Clowe et al. 1998) uses the fact that the shear can be related to a density contrast. More precisely, it can be shown that the following relation holds:

$$
\begin{aligned}
\zeta\left(R_{1}\right) & =\bar{\kappa}\left(<R_{1}\right)-\bar{\kappa}\left(R_{2}<R<R_{\max }\right) \\
& =2 \int_{R_{1}}^{R_{2}} \mathrm{~d} \ln R\left\langle\gamma_{\mathrm{t}}\right\rangle+\frac{2 R_{\max }^{2}}{R_{\max }^{2}-R_{2}^{2}} \int_{R_{2}}^{R_{\max }} \mathrm{d} \ln R\left\langle\gamma_{\mathrm{t}}\right\rangle,
\end{aligned}
$$

where $\bar{\kappa}\left(<R_{1}\right)$ is the mean convergence within a circular aperture of radius $R_{1}$, and $\bar{\kappa}\left(R_{2}<R<R_{\max }\right)$ is the mean convergence in an annulus from $R_{2}$ to $R_{\max }$. This relation shows that the mean surface density within a circle can be derived from the tangential shear profile up to a constant. This is straightforwardly converted into a mass estimate:

$M\left(<R_{1}\right)=\pi R_{1}^{2} \bar{\kappa}\left(<R_{1}\right) \Sigma_{\mathrm{cr}}\left(z_{1}, z_{\mathrm{s}}\right)$.

If wide-field observations are avaliable, $R_{2}$ and $R_{\max }$ can be chosen to be large, so that the surface density in the annulus is negligible. Otherwise, by setting the annulus term to zero, $\zeta$ allows only a lower limit to the projected mass to be estimated.

Although the $\zeta$-statistic would not require any parameterization of the lensing signal to convert the shear into a mass estimate, a complication arises from not directly measuring the shear but the reduced shear. To convert the observed signal into an estimate of the shear, it is usually necessary to make some assumption on the shape of the convergence profile. In our analysis, we follow the method of Hoekstra (2007), who uses the convergence from the best-fit NFW model to the tangential shear profile. We also use this approach to estimate the mean surface density in the annulus. For our mass estimates, $R_{\max }$ varies between $\sim 640$ and $\sim 800$ arcsec, depending on the cluster. We set $R_{2}=0.9 \times R_{\max }$.

\subsection{Strong and weak lensing}

Finally, we consider a completely nonparametric, 2D mass reconstruction. The method used here is not based only on weak lensing. Instead, it combines both strong and weak lensing constraints to provide a map of the lensing potential. The method is fully described in Merten et al. (2009) (see also Bradač et al. 2005, for another similar algorithm). To obtain the underlying lensing potential $\psi$ of the galaxy cluster, the reconstruction algorithm performs a combined $\chi^{2}$-minimization, which consists of a weak and a strong-lensing term, in combination with an additional regularization term:

$\chi^{2}(\psi)=\chi_{\mathrm{w}}^{2}(\psi)+\chi_{\mathrm{s}}^{2}(\psi)+R(\psi)$

The algorithm is grid-based and expresses the derivatives of the lensing potential by finite-differencing schemes. Starting from a coarse initial grid, the resolution is steadily increased until the final reconstruction is reached. At each iteration the reconstruction is regularized on the former iteration by the regularization function $R(\psi)$. This procedure results in a smooth reconstruction of the lensing potential and prevents the reconstruction from following noise patterns in the data.

The weak lensing constraints are obtained by averaging over a certain number of background-galaxy ellipticities in every reconstruction pixel. The error is given by the statistical scatter in each pixel. Afterwards the reduced shear of the cluster is fitted:

$\chi_{\mathrm{w}}^{2}(\psi)=\left(\langle\epsilon\rangle-\frac{Z(z) \gamma(\psi)}{1-Z(z) \kappa(\psi)}\right)_{i} C_{i j}^{-1}\left(\langle\epsilon\rangle-\frac{Z(z) \gamma(\psi)}{1-Z(z) \kappa(\psi)}\right)_{j}$.

The sum over $i$ and $j$ runs over all reconstruction pixels, and the cosmological-weight function $Z(z)$ describes the redshift distribution of the sources

$Z(z) \equiv \frac{D_{\infty} D_{\mathrm{ls}}}{D_{\mathrm{l} \infty} D_{\mathrm{s}}} H\left(z-z_{\mathrm{l}}\right)$,

where $D_{\infty}$ and $D_{\text {lo }}$ are the angular- diameter distances between observer and infinity and between lens and infinity, respectively. We have to solve the full $\chi^{2}$-function since the reconstruction pixels might become correlated on a high grid resolution.

The strong lensing constraints are based on the estimated positions of the critical curves of the galaxy cluster. They are given by the observed arc positions and/or multiple images bracketing the critical lines. Assuming a reconstruction pixel to be part of a critical curve, by summing over all these pixels with index $k$, we find

$\chi_{\mathrm{s}}^{2}(\psi)=\frac{(\operatorname{det} \mathcal{A}(\psi))_{k}^{2}}{\sigma_{\mathrm{s}}^{2}}=\frac{\left((1-Z(z) \kappa(\psi))^{2}-|Z(z) \gamma(\psi)|^{2}\right)_{k}^{2}}{\sigma_{\mathrm{s}}^{2}}$.

The error $\sigma_{\mathrm{s}}$ is determined by the pixelization of the grid.

A final high-resolution step is added, which is able to resolve the positions of the critical curve estimators in greater detail. Since there is no reliable weak lensing information on this resolution, this step is embedded in the foregoing reconstruction by regularizing on the former result in the cluster center:

$\chi_{\text {highres }}^{2}(\psi)=\chi_{\mathrm{s}}^{2}(\psi)+R_{\text {lowres }}(\psi)$.

The mass analysis is then done with a convergence map, which is calculated from the reconstructed lensing potential of the cluster using Eq. (A.5).

In Fig. 8, we show the mass profiles of cluster $g 1-y$ obtained with the weak-lensing methods described above. All the methods perform well by providing consistent results for this particular cluster. The mass profiles deviate from the true one by less than $10 \%$ at all radii. The vertical lines show the position of $R_{2500}, R_{500}$, and $R_{200}$, i.e. the estimated radii enclosing overdensities of 2500,500 , and 200 times the critical density of the 
M. Meneghetti et al.: Weighing simulated galaxy clusters using lensing and X-ray

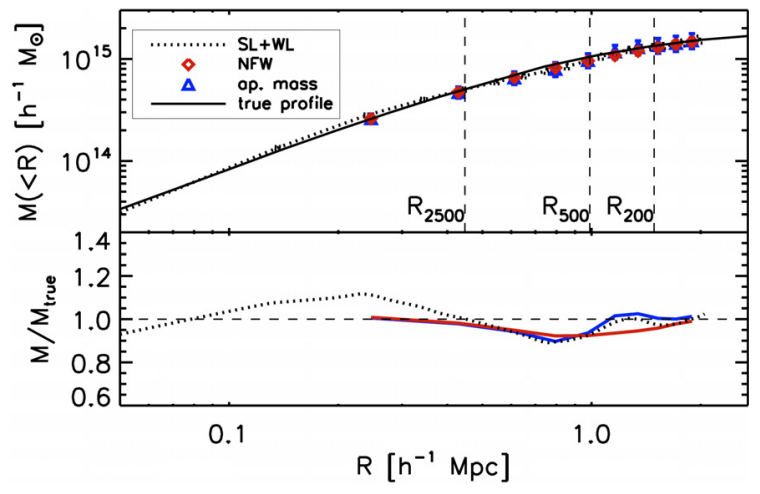

Fig. 8. Results of the weak lensing analysis. Radial 2D-mass profiles of cluster $g 1-y$, as obtained from the three different methods used in this work, namely the NFW fit to the shear profile (diamonds), the aperture mass densitometry (triangles), and the 2D mass reconstruction combining weak and strong lensing (dotted line). The solid line shows the true mass profile. The vertical lines indicate the positions of $R_{2500}$, $R_{500}$, and $R_{2500}$ as derived from the NFW fit. The bottom panel shows the ratios between the mass profiles recovered from the lensing analysis and the true mass profile. The dotted line refers again to the SL+WL method, while the red and blue solid lines indicate the results for the NFW fit and for the aperture mass, respectively.

universe, as derived from the NFW model that best fits the tangential shear profile. In the case of the $2 \mathrm{D}$ mass reconstruction method combining strong and weak lensing, we estimated the errors on the masses by bootstrapping 24 galaxy catalogs and by repeating the reconstruction with each of them. This is computationally very demanding, so we reconstruct the lensing potential a coarse grid of $32 \times 32$ pixels covering the whole cluster field $\left(1280^{\prime \prime} \times 1280^{\prime \prime}\right)$.

\subsection{X-ray}

Soft band [0.7-2] keV X-ray images were created from the event files. With them we identify the regions of dense cold blobs that we mask in any further analysis. The masked regions were overlaid on the X-ray images for each cluster projection in Fig. 4. These bright point-like spots were mostly correlated to the cores of previously merged substructures. Furthermore, we excluded an inner region of $60 \mathrm{kpc} h^{-1}$ for $g 51$ and $70 \mathrm{kpc} h^{-1}$ for $g 1$ and $g 72$ (Fig. 4) to exclude the central region affected by the overcooling problem. From the soft band image, we finally produced the surface brightness profiles using 30 annuli spanning from 23 to 400 arcsec. The surface brightness profile of the cluster $g 1-y$ is given in Fig. 9. In the same radial range, we extraced the spectra of 10-15 annuli logarithmically spaced. We subsequently analyzed the spectra in XSPEC (Arnaud 1996) using a single-temperature MEKAL model (Mewe et al. 1985; Liedahl et al. 1995) to obtain the temperature and the normalization. The values of redshift and hydrogen column density are set accordingly to the input simulation. The temperature profile of $g 1-y$ is shown in Fig. 10.

The X-ray gas and total masses are recontructed using two different methods, here labeled backward and forward methods. In brief, starting from the observed X-ray surface brightness and the radially resolved spectroscopic temperature measurements and under the assumption of spherical geometry and hydrostatic equilibrium between the ICM and the underlying dark matter potential, the backward puts constraints on a parametric functional form of the total mass by deprojecting the observed quantities, while the forward makes direct use of the 3-D model of the gas

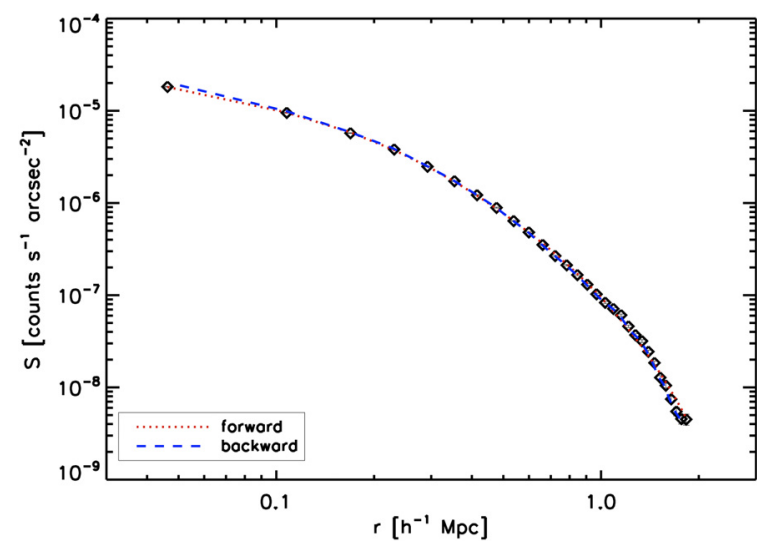

Fig. 9. Radial surface brightness profile of cluster $g 1-y$, as derived from the analysis of the X-ray observation shown in upper middle panel of Fig. 4 (diamonds). The dotted and the dashed lines show the fits to the data obtained with the forward and with the backward methods, respectively.

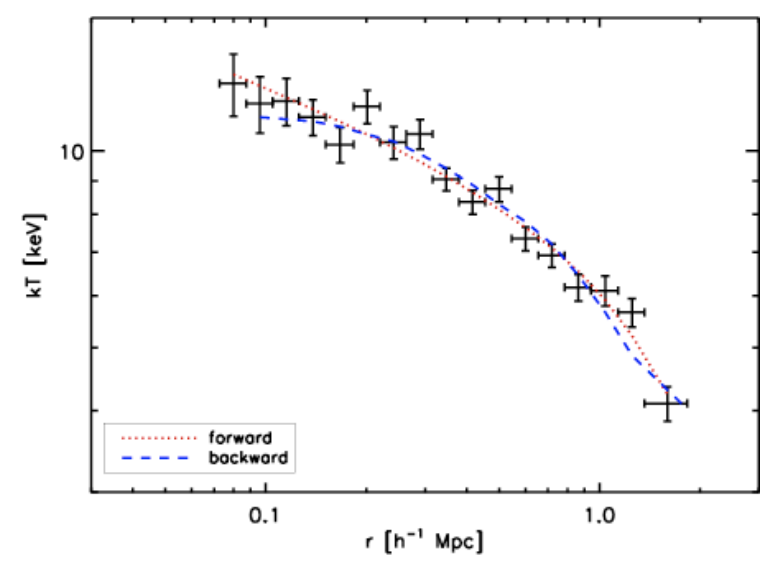

Fig. 10. Radial temperature profile of cluster $g 1-y$, as obtained from the spectral analysis of the X-ray observation (data points). The dotted and the dashed lines show the fits to the data obtained with the forward and with the backward methods, respectively. See text for more details.

density and temperature profiles by estimating the model parameters by projecting the profiles and fitting them to the observed ones.

We describe here the two methods in detail.

Backward method. A functional form of the total mass has to be assumed. From this and moving backward, the profiles of the observed quantities are recovered. In this work, the NFW model in Eq. (2) is used to describe the density profile of the clusters, so that

$M_{\mathrm{tot}}(<r)=4 \pi r_{\mathrm{s}}^{3} \rho_{\mathrm{s}} f(x)$,

where

$f(x)=\ln (1+x)-\frac{x}{1+x}$

and $x=r / r_{\mathrm{s}}$.

The two free parameters $\left(r_{\mathrm{s}}, c_{200}\right)$ are constrained by minimizing a $\chi^{2}$ statistic defined as

$\chi^{2}=\sum_{i} \frac{\left(T_{\text {data }, i}-T_{\text {model }, i}\right)^{2}}{\epsilon_{T, i}^{2}}$ 
where $T_{\text {data }}$ are the either deprojected or observed temperature measurements obtained in the spectral analysis, $T_{\text {model }}$ are the either 3-D or projected values recovered from the inversion of the hydrostatic equilibrium equation for a given gas density and total mass profiles, and $\epsilon_{T}$ is the error on the spectral measurements. The gas density profile, $n_{\text {gas }}$, is estimated from the geometrical deprojection (Fabian et al. 1981; Kriss et al. 1983; McLaughlin 1999; Buote 2000; Ettori et al. 2002) of the measured X-ray surface brightness and, in the present analysis, is used to project $T_{3 \mathrm{D}}$ accordingly to the recipe in Mazzotta et al. (2004) to recover $T_{\text {model }}$. The last values are then compared to the results of the XSPEC analysis using the $\chi^{2}$ statistics (Eq. (25)). The values of $T_{3 \mathrm{D}}$ are obtained from

$-G \mu m_{\mathrm{p}} \frac{n_{\text {gas }} M_{\text {tot }}(<r)}{r^{2}}=\frac{\mathrm{d}\left(n_{\text {gas }} \times T_{3 \mathrm{D}}\right)}{\mathrm{d} r}$,

where $G$ is the gravitational constant, $m_{\mathrm{p}}$ the proton mass, and $\mu=0.59$ the mean molecular weight in a.m.u. as adopted in the present simulations. Further detail on variations and applications of the technique here described are presented in Ettori et al. (2002) and Morandi et al. (2007). The best-fit surface brightness and temperature profiles obtained with this method for the cluster $g 1-y$ are given in Figs. 9 and 10, respectively.

Forward method. By this technique, the total mass profile is recovered through directly (forward) applying the hydrostatic equilibrium equation (see Eq. (26)) once a parametric form of the gas density and temperature profiles hve been estimated. In the following analysis, we adopt the approach by Vikhlinin et al. (2006) that follows similar techniques presented in, e.g., Lewis et al. (2003) and Pratt \& Arnaud (2003) and extends the number of parameters to increase the modeling freedom. In particular, the formula describing the gas density is a $\beta$-model modified to accomodate a power-law behavior in the center and the observed steepening of the surface brightness in the outskirts (Vikhlinin et al. 1999; Neumann 2005; Ettori \& Balestra 2009) with the addition of a second $\beta$-model to better reproduce the core profile:

$$
\begin{aligned}
\rho(r)= & \frac{N_{1}}{\left(r / r_{\mathrm{c} 1}\right)^{-\alpha}\left[1+\left(r / r_{\mathrm{c} 1}\right)^{2}\right]^{\left(3 \beta_{1}-\alpha / 2\right)}} \frac{1}{\left[1+\left(r / r_{\mathrm{s}}\right)^{\gamma}\right]^{(\epsilon / \gamma)}} \\
& +\frac{N_{2}}{\left[1+\left(r / r_{\mathrm{c} 2}\right)^{2}\right]^{3 \beta_{2}}},
\end{aligned}
$$

where $\alpha, \beta_{1}, \beta_{2}, r_{\mathrm{s}}, \epsilon, \gamma, r_{\mathrm{c} 1}$, and $r_{\mathrm{c} 2}$ are all free parameters in the fit. The temperature profile is modeled by a 5 parameter $-T_{0}, r_{\mathrm{t}}, a, b, c-$ function

$T=T_{0} \frac{\left(r / r_{\mathrm{t}}\right)^{-a}}{\left[1+\left(r / r_{\mathrm{t}}\right)^{b}\right]^{c / b}} T_{\mathrm{Cool}}$.

For these simulations, where the cool cores are well inside the excluded inner regions (see Fig. 4), we consider $N_{2}=0$ and $T_{\text {cool }}=1$. These profiles are then projected along the line of sight, considering the Mazzotta et al. (2004) prescription and the best-fit parameters determined from a $\chi^{2}$ minimization technique by comparing the projected quantities with the observed ones. The best-fit surface brightness and temperature profiles for cluster $g 1-y$ are given in Figs. 9 and 10 .

To summarize, by using the observed X-ray surface brightness and temperature profiles, these two methods provide: (backward method) starting from a given gas density profile, the gas mass $M_{\text {gas }}$ and the best-fit parameters $\left(r_{\mathrm{s}}, c_{200}\right)$ from which a total mass profile and a $3 \mathrm{D}$ temperature profiles are recovered; (forward method) for some given parametric forms of the gas and

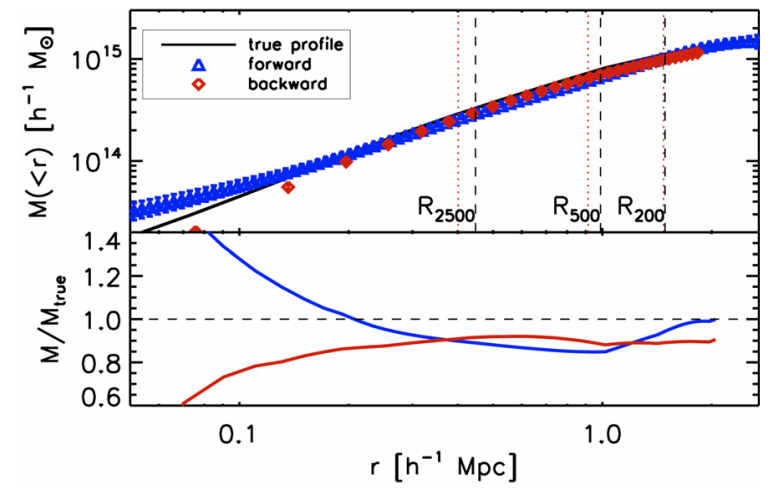

Fig. 11. Results of the X-ray analysis. Radial 3D-mass profiles of cluster $g 1-y$, as obtained from the forward (triangles) and from the backward methods (diamonds) used in this work. The solid line shows the true mass profile. The vertical lines indicate the positions of $R_{2500}, R_{500}$ and $R_{2500}$ as derived from the lensing (see Fig. 8, dashed lines) and from the X-ray backward analyses (dotted lines).

temperature profiles, their best-fit parameters from which $M_{\text {gas }}$ and $M_{\text {tot }}$ are estimated. In the following analysis, the results obtained from these two methods are compared to assess the systematics in the X-ray analysis of the matter distribution.

In Fig. 11 we show the 3D-mass profiles of cluster $g 1-y$, obtained with both the forward and the backward methods. In the region between $R_{2500}$ and $R_{200}$ the two methods are consistent with each other and they underestimate the true mass profile by $\sim 10-20 \%$. Similar results are found for the other clusters in the sample and are discussed in detail in Sect. 4.3. The vertical lines in the figure indicate the estimated sizes of $R_{2500}, R_{500}$, and $R_{200}$. Because of the mass underestimate in the X-ray case, the radii measured through lensing are typically larger than those measured via the X-ray methods. This is clearly shown in Fig. 12 where we report the ratio of the extimated to the true characteristic radii calculated for each cluster projection using the weak lensing signal or the X-ray analysis. It is worth noting that even if the X-ray method always underestimates the true radii, this bias seem to have a quite small scatter. On the contrary while the weak lensing is a less biased estimator, we find for it a much larger scatter. Just for convenience in the following analysis, we compare the X-ray and the lensing masses at the same physical radii, which we choose to be $R_{2500}, R_{500}, R_{200}$ as derived from lensing.

\section{Results}

In this section we discuss the results of the analyses outlined in the previous sections. We start with a discussion of the $2 \mathrm{D}$ mass estimates obtained with the lensing techniques, and then consider the deprojection of the lensing profiles and the X-ray 3Dmass estimates. Finally, we compare lensing and X-ray mass profiles and discuss how our results match the observations.

\subsection{Lensing $2 D$ mass profiles}

\subsubsection{Strong lensing masses}

In Fig. 13, we compare the true and the estimated strong-lensing masses of all clusters in our sample. The 2D masses are measured at the limits of the strong lensing regions, given by the mean distance of the tangential images from the cluster center. The agreement between the estimated and true masses is remarkable, showing that, in most cases, strong lensing methods based 
M. Meneghetti et al.: Weighing simulated galaxy clusters using lensing and X-ray

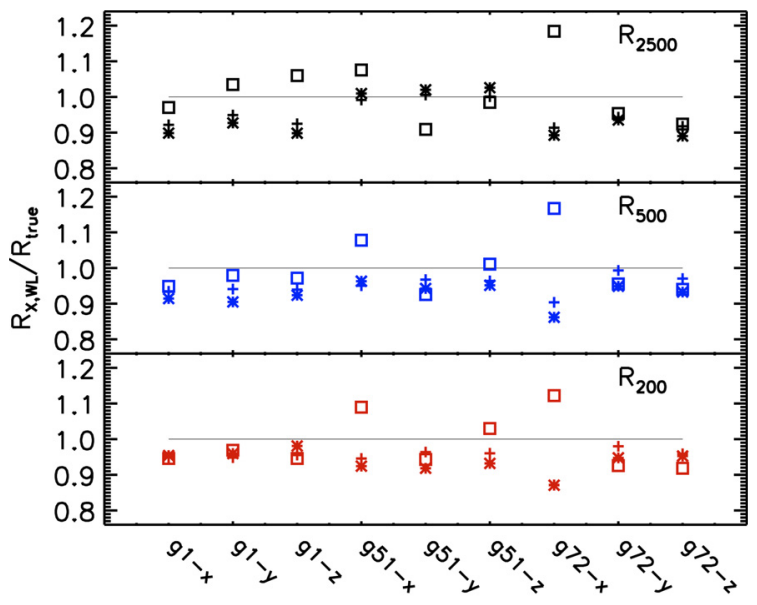

Fig. 12. Ratio of the estimated to the true characteristic radii calculated for each cluster projection. Squares, stars, and crosses refer to size derived using the weak lensing, the X-ray forward, and the X-ray backward method, respectively. The continuous line indicates where the true and the estimated size are equal. The top, middle, and lower panel refer to $R_{2500}, R_{500}$, and $R_{200}$, respectively.

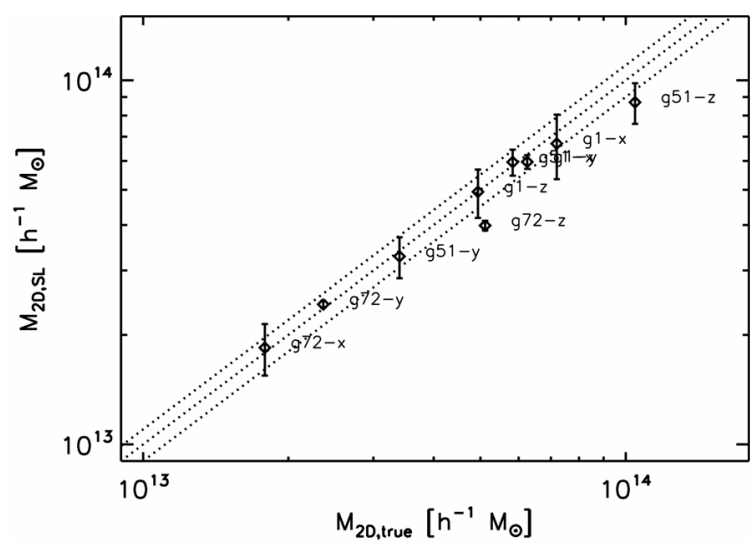

Fig. 13. The projected masses estimated through the strong lensing analysis vs. the corresponding true masses of the lenses. The dotted lines correspond to $M_{2 \mathrm{D}, \mathrm{SL}}=M_{\text {true }}$ and to $M_{2 \mathrm{D}, \mathrm{SL}}=M_{\text {true }} \pm 10 \%$. The masses are measured within a circle centered on the BCG and having a radius equal to the mean distance of the lensing constraints from the cluster center.

on parametric modeling are accurate at the level of a few percent at predicting the projected inner mass. The worst results are obtained for clusters $g 51-z$ and $g 72-z$, for which the offsets between the true and the estimated mass are $\sim 15 \%$ and $\sim 20 \%$, respectively. These two clusters have complex morphologies, as shown in Fig. 1, being characterized by multiple mass components and double cores. For $g 51-z$ the mass model combines 12 mass components, three of which are optimized individually. The cluster $g 72-z$ is modeled with 78 components. In this projection the cluster appears as a double cluster, with two main mass clumps separated by $\sim 50^{\prime \prime}$. Each of these clumps is modeled with an NFW profile and has a massive star concentration at the center, which is modeled as a PIEMD. These four components are optimized individually. Given the complexity of the systems and the large number of free parameters $(24$ for $g 72-z$ ), it is reasonable to expect less accurate mass estimates for these two clusters.

Although the mass measurement is very precise at the position of the tangential critical lines, as anticipated in Sect. 3.1, the extrapolation of strong lensing models to larger radii such

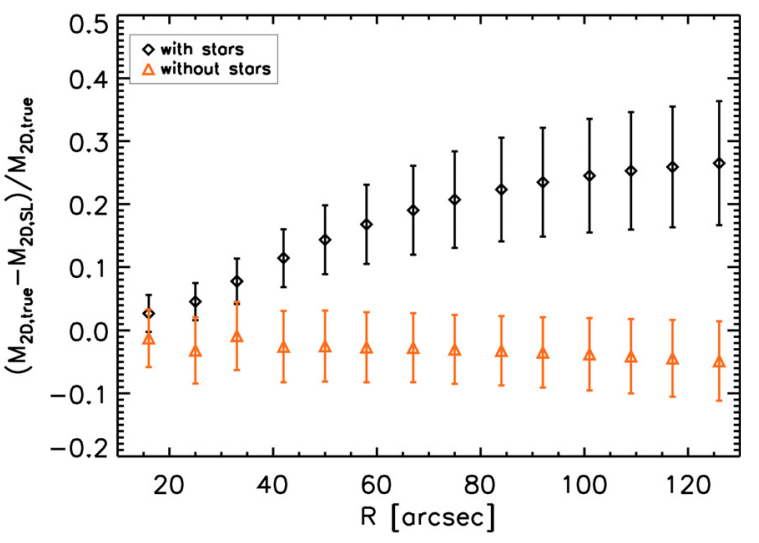

Fig. 14. The relative difference between true and estimated projected masses as a function of the distance from the cluster centers. The results are obtained by averaging over all the clusters in the sample. The diamonds and the triangles refer to the simulations including and excluding the contribution of the BCG to the lensing signal (see text for more details). The errorbars show the scatter among all the reconstructions. The regions probed by SL are typically smaller than $\sim 30-40$ arcsec, thus the mass estimates at larger radii are extrapolations of the SL mass model to distances that are unconstrained by the data.

as $R_{2500}, R_{500}$, or $R_{200}$ could lead to incorrect mass estimates. In particular, we find that the results are very sensitive to the parameterization chosen to model the BCG, when we include the central images in the optimization. Using the wrong model leads to biased mass estimates. In our simulations we adopted a PIEMD, which is a broadly used profile for describing the central galaxies in clusters. This model, however, is not adequate for describing the profile of the BCGs in our simulations. While the PIEMD model is isothermal, i.e. the surface density profile decreases with the distance from the center as $\Sigma_{\mathrm{PIEMD}}(R) \propto R^{-1}$, the true surface density distribution of the stars in our numerical simulations is steeper, i.e. $\Sigma_{\mathrm{BCG}} \propto R^{-1.7}$. This implies that, by fitting the lensing constraints as we do, we require that the BCG is more spatially extended, so an additional contribution to the central mass has to be provided by the dark matter halo. This causes us to systematically overestimate of the halo concentration and underestimate of the scale radius, as shown in Table 3. For this reason, the masses extrapolated to large radii are systematically underestimated. Such a dependence on the BCG mass has been reported recently by Donnarumma et al. (2009) when modeling the cluster MS2137 (see also Comerford et al. 2006). The diamonds in Fig. 14 show the mean relative differences between true and estimated projected masses of the clusters at different distances from the centers. As the distance from the cluster center grows, the amplitude of the bias in the mass estimates increases to about $30 \%$ at a distance of two arcmins. The size of the errorbars reflects the scatter among the reconstructions. The scatter also grows as a function of the distance from the center.

To better illustrate how the results shown above depend on the stellar component of the lenses, we ran a new set of lensing simulations using only the dark matter halos of the clusters as deflectors. Then, we repeated the fitting procedure and we derived the mass profiles as done before, but without modeling the BCGs. In this case, even extrapolating the profiles to radii much larger than the size of the strong lensing region, we find a much smaller disagreement between true and estimated masses. In this case, the strong lensing masses at large radii tend to be on average only slightly higher than the true masses $(<5 \%)$. 

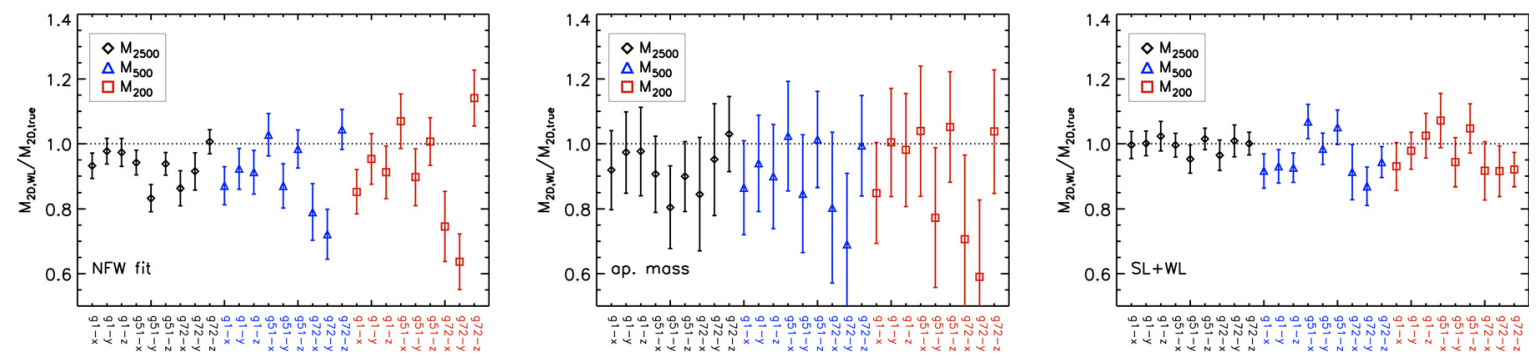

Fig. 15. Comparison between the weak-lensing and the true 2D-masses of all the simulated clusters. From left to right, the panels refer to the methods based on the NFW fit of the shear profile, on the aperture mass densitometry, and on the non-parametric SL+WL reconstruction of the lensing potential, respectively. Shown are the ratios between the estimated and the true masses measured at three characteristic radii, namely $R_{2500}$ (diamonds), $R_{500}$ (triangles), and $R_{200}$ (squares), versus the cluster names.

\subsubsection{Weak lensing masses}

We discuss now the results obtained from the weak-lensing analysis of the clusters in our sample. The accuracy of the mass estimates depends on the morphology of the lenses and on their substructures. No big substructures are contained in the cylinders in the case of cluster $g 1$. As shown in Fig. 1, the cluster appears regular in all three projections. Instead, clusters $g 51$ and $g 72$ have more complex morphologies; in particular, as explained above, $g 72$ has a massive companion $\left(\sim 1.4 \times 10^{14} h^{-1} \mathrm{Mpc}\right)$ located at $\sim 2.5 h^{-1} \mathrm{Mpc}$ from the center. When projected along the $z$-axis of the simulation box, the substructure is very close to the cluster center $\left(\sim 300 h^{-1} \mathrm{kpc}\right)$. For this reason, this mass clump has been included in the strong lensing model of $g 72-z$. Although it lies outside $R_{200}$ in the projections $g 72-x$ and $g 72-y$, this substructure produces a significant shear that complicates the weak lensing mass measurements of the main cluster clump, as described below.

In Fig. 15 we show the ratios of the estimated and of the true $2 \mathrm{D}$-masses of the clusters in the sample. The measurements have been made at $R_{2500}, R_{500}$, and $R_{200}$. The different panels refer to the three methods implemented in this work, namely the NFW fit of the tangential shear profile (left panel), the aperture mass densitometry (central panel), and the SL+WL method (right panel). The reliability of the methods depends strongly on the morphology of the lenses.

Two methods assume that the lensing signal is tangential to the center of the lens, which is assumed to coincide with the BCG. This implies that all shear is assumed to be produced by a main cluster clump. These are the NFW fit and the aperture mass densitometry methods. For some lenses, such as the three projections of $g 1$, this approximation is fairly well met. Indeed, this cluster is free of large substructures, and the shear is dominated by the main cluster clump. For some other lenses, such as the three projections of $g 72$, this is not the case. In all three projections of this cluster, the shear produced by the secondary mass clump contributes significantly to the total shear. As a result, the tangential shear with respect to cluster center is smaller than it should be in the projection along the $x$ - and the $y$-axes in the absence of the secondary mass component. When fitting the shear profile with a single NFW model or converting the shear into a mass estimate using the aperture mass densitometry, this causes to underestimate the mass. The amplitude of this effect grows with increasing distance from the cluster center, because the secondary mass clump is approached. In the case of $g 72-x$ and $g 72-y$, using the NFW fit method, the mass is underestimated by $\sim 10-15 \%$ at $R_{2500}$ and by $\sim 30-40 \%$ at $R_{200}$. Similar results are found with the aperture mass densitometry. In the case of $g 72-z$, where the secondary clump is located near the cluster center, the shear produced by the main cluster and by the substructure sum up. The resulting tangential shear mimics that of a lens with a higher mass and with an extended core, because of the offset between the two mass clump centers. As a result, we find that the estimated $M_{200}$ exceeds the true value by $\sim 15-20 \%$.

To validate our interpretation of these results, we performed the following tests. First, we placed two NFW halos of mass $M_{1}=6.8 \times 10^{14} h^{-1} M_{\odot}$ and $M_{2}=1.4 \times 10^{14}$ at a distance of $2.5 \mathrm{~h}^{-1} \mathrm{Mpc}$, and we calculated the shear field produced by these two mass clumps. Then, we sampled the shear field at random galaxy positions and added the noise due to the intrinsic galaxy ellipticities, following the method outlined in Maturi et al. (2005). Finally we measured the tangential shear profile with respect to the center of the most massive halo and fit it using a single NFW profile, over a radial range similar to the one used in our numerical simulations. We repeated the experiment after removing the least massive halo. We find that, if the shear produced by the second halo is included in the simulation, the masses at $R_{2500}, R_{500}$, and $R_{200}$ are underestimated with respect to the simulation including only the most massive halo, by $4 \%$, $21 \%$, and $30 \%$, respectively. This qualitatively agrees with the results for $g 72-x$ and $g 72-y$. In both cases, the tangential shear profile is well-fitted by an NFW profile, i.e. with reduced $\chi^{2} \lesssim 1$. As a second test, we placed the least massive halo at a distance of $300 h^{-1} \mathrm{kpc}$ from the main halo. The resulting tangential shear profile is compared with the one produced by a single-mass component of total mass $M=M_{1}+M_{2}$. By fitting with NFW models, we find that, in the two-halo case, the masses at $R_{2500}, R_{500}$, and $R_{200}$ are overestimated by $3 \%, 17 \%$, and $25 \%$, respectively, with the respect to the single-halo case. This is also in good agreement with our results for $g 72-z$.

For the remaining clusters, the mass estimates obtained with these two methods are more accurate. Typically, the estimated masses deviate by $\$ 15 \%$ from the true masses. Even in the case of $g 51$, despite the presence of a few substructures in the cluster surroundings, the mass estimates are in good agreement with the input masses. The reason is that the above-mentioned substructures are less massive than in the case of $g 72$ and the shear is dominated by the cluster halo.

The nonparametric SL+WL method does not require any assumption about the symmetry of the lensing signal. Substructures are included in the mass model by construction. For this reason, the SL+WL method can recover the input mass with good precision even in the case of morphologically disturbed clusters. We find that the deviations between estimated and true masses are typically below the $10 \%$ level.

For all the three methods, the scatter between estimated and true masses grows as a function of the radius, in agreement with Okabe et al. (2009). 
M. Meneghetti et al.: Weighing simulated galaxy clusters using lensing and X-ray
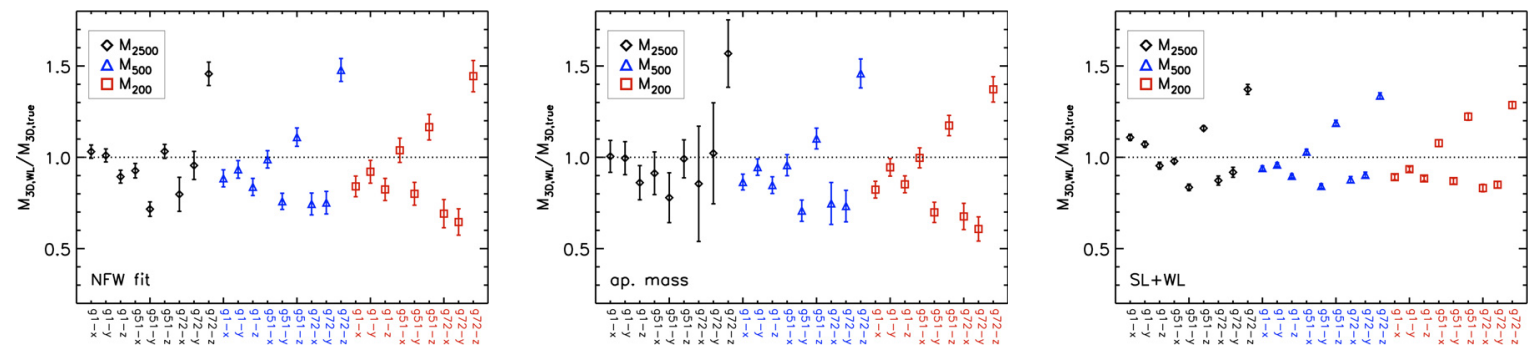

Fig. 16. As in Fig. 15, but comparing the estimated and true 3D-masses.

\subsection{Lensing 3D masses}

As stated several times above, lensing measures the total mass contained in cylinders and projected on the sky. To convert the 2D-mass into 3D-mass estimates, deprojection needs to be implemented. This requires to make some assumptions on the shape of the clusters and on their 3D density profile. We assume here that clusters are spherical and that their density profile is well described by the NFW model.

Deprojection is done differently for the three methods investigated here. For the NFW fit method, we use the NFW parameters obtained from the fit of the tangential shear profile to calculate the 3D-mass profile of the lenses. For the aperture mass densitometry and for the SL+WL methods, we fit the 2D-mass profiles with projected NFW models and we use the best-fit parameters to derive the 3D-mass profiles. These 3D-profiles are shown in Fig. B.1 for the individual cluster projections.

Similar to Fig. 15, we show in Fig. 16 the ratios between estimated and true 3D-masses at three over-density radii. It is clear that in $3 \mathrm{D}$ the scatter between the estimates and the input masses is significantly larger than in 2D. This is due to two main reasons. First, lenses are triaxial, while we are assuming spherical symmetry during the deprojection. The impact of triaxiality on lensing mass estimates was already highlighted by several authors in past (e.g. Gavazzi 2005; Oguri et al. 2005). Depending on the degree of triaxiality and on the orientation of the clusters with respect to the line of sight, 3D-masses may result to be overor under-estimated. In particular, we find that, in the cases of good alignment (i.e. small angles) between the major axis of the cluster and the projection axis, the lensing masses tend to be systematically larger than the true masses, while the opposite occurs in those cases where the major axis is nearly perpendicular to the line of sight. This is shown in Fig. 17, where the lensing masses are derived with the SL+WL method, which even in 3D seems to provide the most accurate mass estimates. Given that the masses of ellipsoids and spheres with the same azimuthal density profile tend to converge at large distances from their centers, the effect is strongest for $M_{2500}$ and for $M_{500}$, and mildest for $M_{200}$. However, even at $R_{200}$, the analysis of our sample shows that the scatter due to triaxiality is $\sim 20 \%$. Similar results are found by Corless \& King (2007).

The second factor which makes the 3D lensing mass estimates so noisy is the presence of substructures along the line of sight. Since their distance from the lens plane is unknown, the 3D-mass estimates can be severely affected by these mass clumps, especially if they are located close to the cluster core in projection. The high ratio between the estimated and the true mass of $g 72-z$ is in large part due to the presence of the massive sub-clump previously mentioned in the paper. This accounts for $\sim 15 \%$ of the total cluster mass, but its erroneous inclusion in the central $300 h^{-1} \mathrm{kpc}$ significantly affects the mass estimates at small radii.

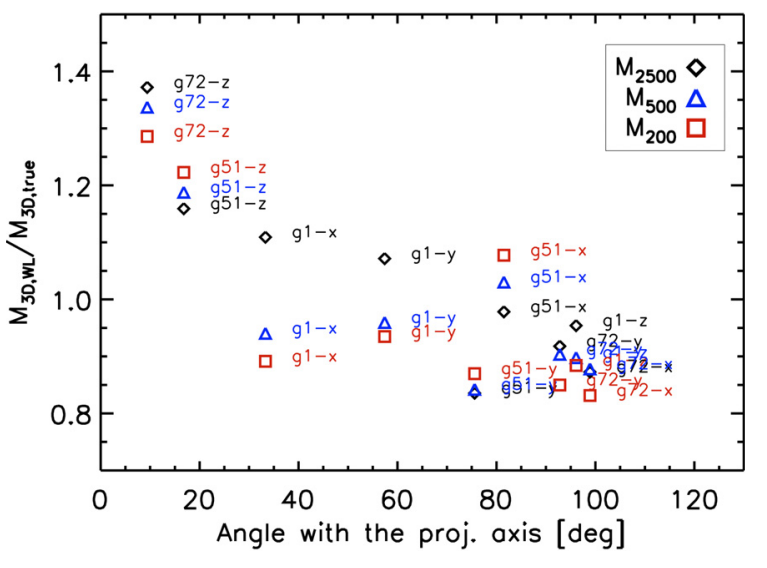

Fig. 17. Ratio between estimated and true lensing masses as a function of the angle between the major axis of the cluster inertia ellipsoid and the axis along which the mass distribution is projected. The results are shown for the lensing masses obtained with the SL+WL method. Squares, triangles and diamonds indicate the mass measurements at $R_{200}, R_{500}$, and $R_{2500}$, respectively.

Apart from this particular cluster projection, in the cases of systems without large substructures along the line of sight, 3D-lensing masses are affected by an intrinsic uncertainty due to triaxiality, which we estimate to be about $20 \%$ at $R_{200}$. Unfortunately, at smaller radii where the lensing measurements of the $2 \mathrm{D}$ masses would be more robust, the scatter becomes larger, since it is around $50 \%$.

Recently by studying the weak lensing signal of 30 galaxy clusters observed with the SUBARU telescope, Okabe et al. (2009) have found that the mean ratios between 3D- and 2D-masses at $R_{\mathrm{vir}}$ and $R_{500}$ are $1.34 \pm 0.17$ and $1.40 \pm 0.10$, respectively, where $R_{\text {vir }}$ is the virial radius. They derive the $2 \mathrm{D}-$ masses using the aperture mass densitometry method, while the 3D-masses are obtained from the NFW fits of the shear profiles. Their sample spans a range of masses that is much wider than covered by our sample. From their Fig. 8, we can estimate that, by limiting the analysis to masses $M_{\mathrm{vir}} \geq 8 \times 10^{14} h^{-1} M_{\odot}$ and $M_{500} \geq 4 \times 10^{14} h^{-1} M_{\odot}$, the ratios are lower $(\sim 1.06$ and $\sim 1.37$ at $R_{\text {vir }}$ and at $R_{500}$, respectively). Averaging over our sample, we find $M_{\mathrm{vir}, 2 \mathrm{D}}^{\mathrm{ap}} / M_{\mathrm{vir}, 3 \mathrm{D}}^{\mathrm{NFW}}=1.14 \pm 0.09$ and $M_{500,2 \mathrm{D}}^{\mathrm{ap}} / M_{500,3 \mathrm{D}}^{\mathrm{NFW}}=1.29 \pm$ 0.08 . These are in quite good agreement with the the ratios between the true 2D- and 3D-masses, which are $M_{\text {vir,2D }}^{\text {true }} / M_{\text {vir,3D }}^{\text {true }}=$ 1.13 and $M_{500,2 \mathrm{D}}^{\text {true }} / M_{500,3 \mathrm{D}}^{\text {true }}=1.34$. From these results, we can deduce that a) on average, the ratios between 2D- and 3D-masses are well recovered, despite triaxiality and substructures affecting individual mass estimates, and b) the agreement between simulations and observations is an indication that the density profiles of real and simulated clusters are, within the errors, compatible with each other. 

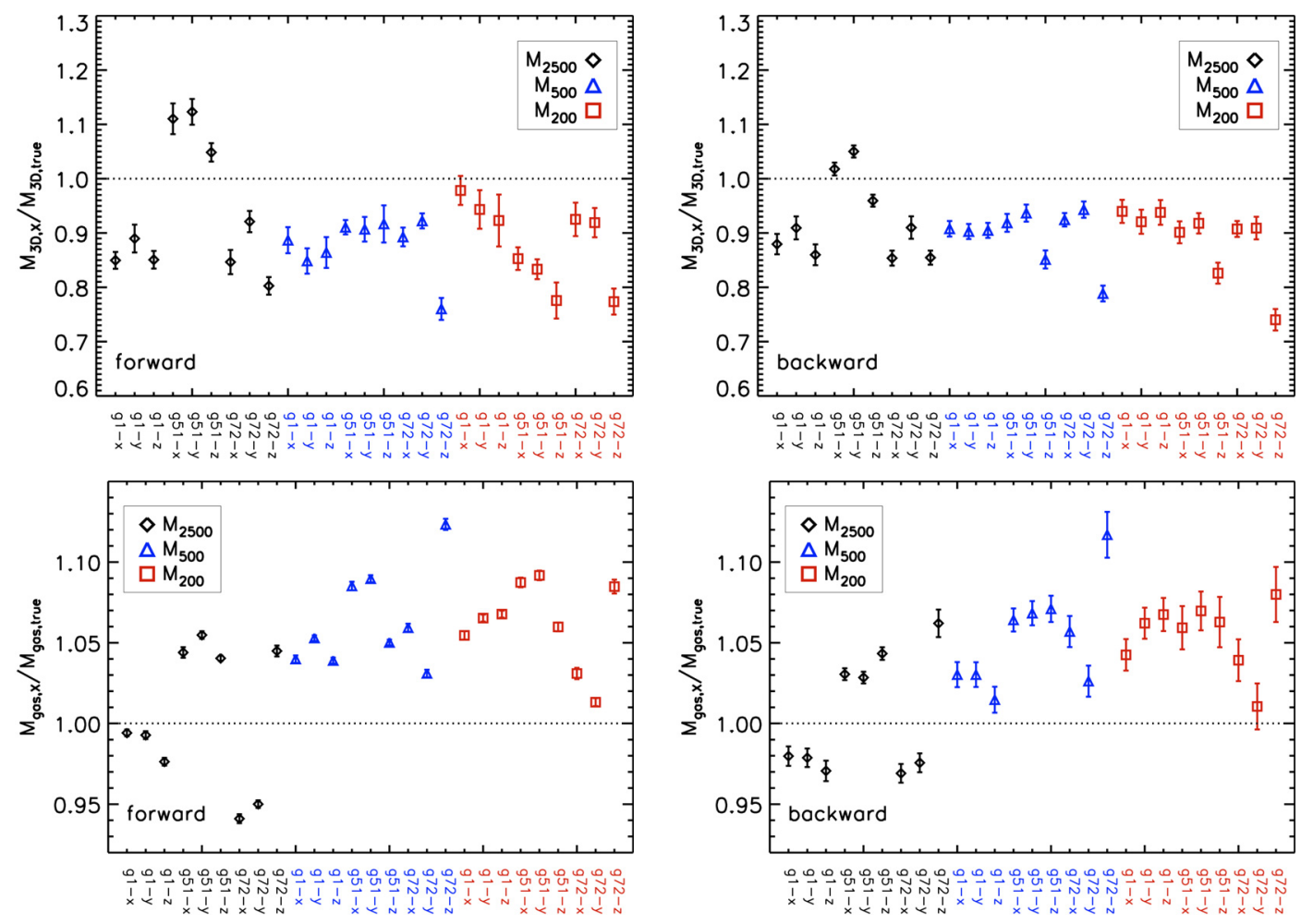

Fig. 18. Top panels: comparison between the X-ray and the true 3D-masses of all the simulated clusters. The left and the right panels refer to the forward and backward methods, respectively. Shown are the ratios between the estimated and the true masses measured at three characteristic radii, namely $R_{2500}$ (diamonds), $R_{500}$ (triangles), and $R_{200}$ (squares), as a function of the cluster name. Bottom panels: as for the upper panels, but comparing the gas masses derived from the X-ray analysis to the true gas masses of the clusters.

\subsection{X-ray masses}

In Fig. 18 we compare the true and estimated total (dark matter and gas) and gas only masses for all the cluster in our sample. The left and the right panels refer to the forward and to the backward methods described in Sect. 3.4. The top panels show the ratios between the estimated and the true masses measured at three characteristic radii, namely $R_{2500}$ (diamonds), $R_{500}$ (triangles), and $R_{200}$ (squares), as a function of the cluster name. A comparison of the mass profiles at any other radius is shown in Fig. B.1. The forward and backward methods give results which, at the three different characteristic radii, are consistent within $2 \sigma$ for all the clusters. The reconstruction of the X-ray masses through the two methods can differ because, while the backward method assumes a functional form of the total density profile imposing the gas temperature profile for any given gas density profile, the forward method reproduces the gas density and temperature with very flexible models that, in particular in the outer regions, can induce unstable extrapolations.

For the forward method, the confidence intervals on mass profiles have been estimated by minimizing the distance between the projected models and a set of 100 random realizations of observed profiles. These profiles were obtained assuming Gaussian statistics around observed values of the surface brightness and temperature profiles with the constraint of rejecting realizations leading to non-physical solution of non-monotonically increasing mass profiles. For the backward method, errors on $M_{\text {gas, } \mathrm{X}}$ are estimated by propagation of the errors on the gas density profile as obtained from the direct deprojection of the surface brightness, reconstructed 100 times by considering the Gaussian error in each radial bin. The error on $M_{3 \mathrm{D}, \mathrm{X}}$ is obtained from the propagation of the errors on the concentration parameter and scale radius estimated through Eq. (25).
Similar to what is shown in other work (see e.g. Rasia et al. 2006, 2008; Nagai et al. 2007, and references therein), we find that, overall the X-ray mass estimates are biased low by $5 \%-20 \%$. More quantitatively we find that, on average, both methods underestimate the true mass by $10 \%$ with a scatter of $6 \%$ almost independently of the characteristic radius considered. The most deviant mass estimate comes only from the $z$ projection of cluster $g 72$. This again comes from this cluster being composed by two structures that line up along the line of sight (see Fig. 1). When we fit the surface brightness profile this appears to be steeper in the center and shallower at the characteristic radius.

In the bottom panels of Fig. 18, we compare the gas masses derived from the X-ray analysis to the true gas masses of the clusters. Again, we find that the two methods give consistent results within $2 \sigma$ errors. Furthermore, in agreement with other work, we find that the total gas mass is fully reconstructed (Nagai et al. 2007). More quantitatively we find that, on average, the gas mass is recovered to better than $1 \%$ with a $3 \%$ scatter at $R_{2500}$ and $7 \%$ with a $3 \%$ scatter at $R_{500}$ and $R_{200}$. It is important to say that the trend to slightly overestimate the gas mass at large radii comes from both X-ray methods tending to slightly overestimate the cluster density profiles in the outskirts due to the imperfect azimuthal symmetry of the cluster surface brightness at larger radii. The most deviant gas mass estimates is given by the $z$ projection of cluster $g 72$. Again this results from the same effect explained above for the mass.

\subsection{NFW fit parameters}

The best-fit NFW parameters for all the clusters in the sample, obtained both through the lensing and X-ray analyses in the 
M. Meneghetti et al.: Weighing simulated galaxy clusters using lensing and X-ray

Table 2. Radial ranges used for fitting NFW profiles to the lensing and X-ray data, with all radii expressed in units of $r_{200}$ reported in Table 1 .

\begin{tabular}{lcccc}
\hline \hline Cluster & Min-max SL & Min-max WL & Min-max SL+WL & Min-max X-ray \\
\hline$g 1-x$ & $0.002-0.08$ & $0.16-1.00$ & $0.03-1.00$ & $0.45-0.90$ \\
$g 1-y$ & $0.002-0.09$ & $0.16-1.00$ & $0.03-1.00$ & $0.45-0.90$ \\
$g 1-z$ & $0.002-0.06$ & $0.16-1.00$ & $0.03-1.00$ & $0.45-0.90$ \\
$g 51-x$ & $0.003-0.07$ & $0.20-1.00$ & $0.05-1.00$ & $0.50-1.00$ \\
$g 51-y$ & $0.002-0.05$ & $0.20-1.00$ & $0.05-1.00$ & $0.50-1.00$ \\
$g 51-z$ & $0.002-0.12$ & $0.20-1.00$ & $0.05-1.00$ & $0.50-1.00$ \\
$g 72-x$ & $0.006-0.05$ & $0.20-1.00$ & $0.05-1.00$ & $0.54-1.08$ \\
$g 72-y$ & $0.006-0.05$ & $0.20-1.00$ & $0.05-1.00$ & $0.54-1.08$ \\
$g 72-z$ & $0.002-0.05$ & $0.20-1.00$ & $0.05-1.00$ & $0.54-1.08$ \\
\hline
\end{tabular}

Table 3. The NFW concentrations and scale radii (upper and lower part of the table, respectively) resulting from the strong-lensing (Col. 4), weak-lensing (Col. 5), strong+weak lensing (Col. 6), and X-ray analyses (Cols. 7, 8 for the forward and for the backward methods) of the clusters in our sample.

\begin{tabular}{|c|c|c|c|c|c|c|c|}
\hline Cluster & $c_{\text {true }}^{\text {DMonly }}$ & $c_{\text {true }}^{\text {total }}$ & $c^{\mathrm{SL}}$ & $c^{\mathrm{WL}}$ & $c^{\mathrm{SL}+\mathrm{WL}}$ & $c^{\mathrm{X}, \text { forw }}$ & $c^{\mathrm{X}, \mathrm{back}}$ \\
\hline$g 1-x$ & 4.62 & 5.38 & $11.31_{-059}^{0.19}$ & $6.21 \pm 0.87$ & $6.59 \pm 0.32$ & $3.58^{0.06}$ & $3.59^{0.32}$ \\
\hline$g 1-y$ & 4.62 & 5.38 & $10.57_{-181}^{2.82^{29}}$ & $4.82 \pm 0.64$ & $5.39 \pm 0.23$ & $4.51_{-0.08}^{0.086}$ & $4.05_{-0.25}^{0.40}$ \\
\hline$g 1-z$ & 4.62 & 5.38 & $6.92_{-1.12}^{2.25}$ & $4.44 \pm 0.62$ & $4.54 \pm 0.23$ & $4.06_{-0.07}^{0.078}$ & $3.62_{-0.31}^{0.22^{5}}$ \\
\hline$g 51-x$ & 5.37 & 7.20 & $3.36_{-0.11}^{0.46}$ & $5.83 \pm 0.96$ & $6.10 \pm 0.29$ & $11.15_{-0.020}^{0.079}$ & $10.76_{-0.06}^{0.31}$ \\
\hline$g 51-y$ & 5.37 & 7.20 & $9.72_{-0.61}^{0.68}$ & $5.13 \pm 1.05$ & $6.35 \pm 0.42$ & $12.39^{0.22} 0_{-022}^{-.20}$ & $10.80_{-0.56}^{0.0 .65}$ \\
\hline$g 51-z$ & 5.37 & 7.20 & $8.78_{-0.46}^{0.61}$ & $6.06 \pm 0.93$ & $7.41 \pm 0.32$ & $11.04_{-022}^{0.2122}$ & $11.30_{-0.02}^{0.0 .56}$ \\
\hline$g 72-x$ & 3.99 & 4.22 & $6.46_{-2.54}^{0.99}$ & $4.17 \pm 1.54$ & $3.88 \pm 0.30$ & $3.31_{-0.05}^{0.0522}$ & $3.22_{-0.02}^{0.06}$ \\
\hline$g 72-y$ & 3.99 & 4.22 & $7.26_{-0.14}^{1.57^{4}}$ & $7.91 \pm 2.86$ & $4.17 \pm 0.31$ & $3.48^{0.05}$ & $3.29^{0.062}$ \\
\hline$g 72-z$ & 3.99 & 4.22 & $11.39_{-0.85}^{0.42}$ & $4.19 \pm 0.62$ & $4.50 \pm 0.24$ & $4.92_{-0.08}^{0.085}$ & $4.49_{-0.29}^{0.082}$ \\
\hline$c / c_{\text {true }}^{\text {DMonly }}$ & & & $1.84(0.60)$ & $1.17(0.30)$ & $1.15(0.15)$ & $1.33(0.59)$ & $1.25(0.56)$ \\
\hline $\begin{array}{l}c / \text { true } \\
c / c_{\text {true }}^{\text {total }}\end{array}$ & & & $1.59(0.59)$ & $1.01(0.33)$ & $0.98(0.11)$ & $1.09(0.38)$ & $1.03(0.36)$ \\
\hline$c / c_{\text {true }}^{\text {DMonly }}$ & no $g 51$ & & $2.09(0.48)$ & $1.23(0.35)$ & $1.12(0.15)$ & $0.92(0.15)$ & $0.86(0.12)$ \\
\hline \multirow[t]{2}{*}{$c / c_{\text {true }}^{\text {ctot }}$} & no $g 51$ & & $1.88(0.45)$ & $1.12(0.35)$ & $1.00(0.12)$ & $0.83(0.15)$ & $0.78(0.13)$ \\
\hline & $r_{\mathrm{s}, \text { true }}^{\text {DMonly }}$ & $r_{\mathrm{s}, \text { true }}^{\text {total }}$ & $r_{\mathrm{s}}^{\mathrm{SL}}$ & $r_{\mathrm{s}}^{\mathrm{WL}}$ & $r_{\mathrm{s}}^{\mathrm{SL}+\mathrm{WL}}$ & $r_{\mathrm{s}}^{\mathrm{X}, \text { forw }}$ & $r_{\mathrm{s}}^{\mathrm{X}, \text { back }}$ \\
\hline$g 1-x$ & 0.310 & 0.278 & $0.097_{-0.002}^{0.007}$ & $0.229 \pm 0.037$ & $0.221 \pm 0.012$ & $0.408_{-0.007}^{0.007}$ & $0.409^{0.038}$ \\
\hline$g 1-y$ & 0.310 & 0.278 & $0.090_{-0.006}^{0.0602}$ & $0.307 \pm 0.048$ & $0.276 \pm 0.013$ & $0.315_{-0.006}^{0.006 /}$ & $0.360_{-0.036}^{0.026}$ \\
\hline$g 1-z$ & 0.310 & 0.278 & $0.152_{-0.031}^{0.0296}$ & $0.317 \pm 0.053$ & $0.320 \pm 0.017$ & $0.351_{-0.07}^{0.0006}$ & $0.403_{-0.027}^{0.0436}$ \\
\hline$g 51-x$ & 0.241 & 0.189 & $0.385_{-0.053}^{0.0731}$ & $0.242 \pm 0.045$ & $0.235 \pm 0.012$ & $0.120_{-0.002}^{0.0007}$ & $0.124_{-0.006}^{0.0097}$ \\
\hline$g 51-y$ & 0.241 & 0.189 & $0.099_{-0.012}^{0.0053}$ & $0.246 \pm 0.057$ & $0.206 \pm 0.014$ & $0.107_{-0.002}^{0.002}$ & $0.124_{-0.007}^{0.0086}$ \\
\hline$g 51-z$ & 0.241 & 0.189 & $0.141_{-0.003}^{0.0012}$ & $0.244 \pm 0.043$ & $0.204 \pm 0.010$ & $0.120_{-0.003}^{0.0002}$ & $0.116_{-0.007}^{0.008}$ \\
\hline$g 72-x$ & 0.299 & 0.299 & $0.087_{-0.009}^{0.032}$ & $0.262 \pm 0.105$ & $0.306 \pm 0.025$ & $0.366_{-0.006}^{0.0003}$ & $0.386_{-0.007}^{0.001}$ \\
\hline$g 72-y$ & 0.299 & 0.299 & $0.094_{-0.027}^{0.0299}$ & $0.135 \pm 0.053$ & $0.287 \pm 0.023$ & $0.355^{0.0005}$ & $0.386_{-0.008}^{0.002}$ \\
\hline$g 72-z$ & 0.299 & 0.299 & $0.059_{-0.004}^{0.0027}$ & $0.367 \pm 0.062$ & $0.324 \pm 0.018$ & $0.223_{-0.004}^{0.0006}$ & $0.251_{-0.022}^{0.0 .019}$ \\
\hline$r_{\mathrm{s}} / r_{\mathrm{s}, \text { true }}^{\text {DMonly }}$ & & & $0.49(0.40)$ & $0.93(0.20)$ & $0.93(0.11)$ & $0.89(0.33)$ & $0.97(0.36)$ \\
\hline$r_{\mathrm{s}} / r_{\mathrm{s}, \text { true }}^{\text {totala }}$ & & & $0.59(0.53)$ & $1.05(0.27)$ & $1.04(0.12)$ & $0.98(0.32)$ & $1.06(0.34)$ \\
\hline$r_{\mathrm{s}} / r_{\mathrm{s}, \text { true }}^{\text {DMly }}$ & no $g 51$ & & $0.31(0.09)$ & $0.88(0.24)$ & $0.95(0.12)$ & $1.10(0.18)$ & $1.20(0.17)$ \\
\hline$r_{\mathrm{s}} / r_{\mathrm{s}, \mathrm{trua}}^{\mathrm{totue}}$ & no $g 51$ & & $0.34(0.11)$ & $0.94(0.26)$ & $1.001(0.11)$ & $1.17(0.22)$ & $1.27(0.21)$ \\
\hline
\end{tabular}

radial ranges listed in Table 2, are summarized in Table 3. In Cols. 2 and 3, we quote the true concentrations obtained by fitting the DM-only and the total density profiles of the three clusters in the radial range between $10 h^{-1} \mathrm{kpc}$ and $r_{200}$. Below each column, we report the mean ratios of the estimated and the true parameters. These are calculated both including and neglecting the three projections of 951 , which strongly bias the mean concentrations and scale radii derived from the X-ray analyses, as discussed later in the text. The numbers in parentheses are the corresponding rms values.

In Col. 4 of the table we report the best-fit concentrations and scale radii obtained for the main cluster halo using the stronglensing constraints. These results were obtained by including the central images in the strong lensing modeling. As discussed in Sect. 4.1.1, this leads to systematically overestimating the concentration and underestimating the scale radius, being the PIEMD model used in the SL modeling inadequate to describe the distribution of the stars in the simulations. In particular, we note that, in some cases, the concentrations are off by a factor of $\sim 3$ with respect to the true values reported in the second column. On average, the concentrations derived from SL alone are almost $85 \%$ higher than the true concentrations of the DM-only profiles. Conversely, the scale radii are almost $\sim 50 \%$ lower. We recall that the SL fits refer to the cluster halos, while the stars are modeled apart. Such a bias is not present in the simulations without stars, where the average ratio between estimated and true concentrations is around unity.

The weak-lensing best-fit parameters, obtained from the fit of the shear profiles, are given in Col. 5. The concentrations tend to be slightly higher than those of the dark-matter 
distributions in the input models $\left(\left\langle c^{\mathrm{WL}} / c_{\text {true }}^{\text {DMonly }}\right\rangle=1.17\right)$ and the scale radii are on average $\sim 10 \%$ smaller than the input values $\left(\left\langle r_{\mathrm{s}}^{\mathrm{WL}} / r_{\mathrm{s}, \text { true }}^{\mathrm{DM}}\right\rangle=0.93\right)$. Similar results are found by combining SL and WL (Cols. 6). In this case the average ratio between estimated and true concentrations is $\left\langle c^{\mathrm{SL}+\mathrm{WL}} / c_{\text {true }}^{\text {DMonly }}\right\rangle=1.15$. For the scale radius we find $\left\langle r_{\mathrm{s}}^{\mathrm{SL}+\mathrm{WL}} / r_{\mathrm{s} \text {,true }}^{\mathrm{DMonl}}\right\rangle=0.93$. The tendency to overestimate the concentration more when adding the SL constraints is caused by the large contribution of the stellar and gas masses within the inner $100 h^{-1} \mathrm{kpc}$, as shown in Fig. 2. We recall that in the cases of the WL and WL+SL methods the fits are done over the total projected mass profiles, thus without distinguishing the dark-matter component from the stellar and gas masses. To support this interpretation, we note that the largest discrepancies with the true concentrations arise for the cluster 951 , which is characterized by an extended strong over-cooling region in the center, where the total density profile steepens compared to the DM only profile. This mimics a higher concentration, because the mass profile still compatible with an NFW model. Fitting the total density profiles of the inputs clusters indeed leads to higher concentrations and smaller scale radii, as reported in Col. 3, which are in a much better agreement with the results of the lensing fits. As highlighted in the previous sections, the WL fits of some systems, like the three projections of $g 72$, can be strongly biased when assuming a single mass component. Indeed, their substructures need to be properly modeled when deriving the cluster mass from the shear signal. The SL+WL method provides a better chance to measure the density profile, at least in some cases, like $g 72-x$ and $g 72-y$, where the large substructure lying at the edge of the cluster virial region is by construction properly modeled. For $g 72-z$, where the substructure is near the cluster core, a correct fit of the projected mass distribution would also require multiple mass components for the SL+WL method.

Comparable estimates of $c$ and $r_{\mathrm{s}}$ are obtained with the two $\mathrm{X}$-ray techniques. Even the X-ray analysis probes the total mass distribution, including the gas and the stars. We note that, using the backward method, the concentration and the scale radius are obtained as best-fit parameters that minimize the reconstructed temperature profile with respect to the observed values, whereas using the forward method let us estimate them with a-posteriori fit with a NFW functional form performed in the radial range $0.7-1.4 h^{-1} \mathrm{Mpc}$ on the mass model obtained by applying the hydrostatic equilibrium equation to the gas density and temperature models described in Eqs. (27) and (28), respectively. Overall, the deviations from the true estimates go in the same direction: in $g 1$, $c$ is underestimated consistently by a factor $0.7-0.8$, with a corresponding overestimate of $r_{\mathrm{s}}$ up to a factor 1.45, if compared with the true concentrations obtained by fitting the input total mass distributions of the clusters. The same considerations apply to $g 72$, apart from $g 72-z$, where the concentration (scale radius) is estimated higher (lower) than $c_{\text {true }}^{\text {total }}\left(r_{\text {s,true }}^{\text {total }}\right)$ by about 15 per cent as a consequence of the alignment along the line of sight of the two main clumps. More critical is the case of $g 51$, where both the X-ray methods provide a measure of the concentration that is twice $c_{\text {true }}^{\text {total }}$, again because of the large contribution of the cool substructures in the central regions, as also shown from the overestimate of the total mass obtained through X-ray analysis within $R_{2500}$ (see Fig. 18). Although we attempted to mask it in the $\mathrm{X}$-ray analysis, the over-cooling region in this cluster is very extended, thus it is still affecting the mass reconstruction (see Fig. B.1 in Appendix B). On average the X-ray concentrations are $\sim 10 \%$ and $\sim 20 \%$ lower than $c_{\text {true }}^{\text {DMonly }}$ and $c_{\text {true }}^{\text {total }}$, respectively, if the three projections of $g 51$ are not included.

\subsection{Lensing vs. X-ray $3 D$ mass profiles}

Finally, we attempted a comparison between the lensing and the $\mathrm{X}$-ray mass estimates. First of all, given the results discussed above, it is not surprising that lensing and X-ray mass estimates in individual galaxy clusters can differ by up to $100 \%$. This is clear in Fig. 19, where the lensing masses are shown as a function of their X-ray equivalents. The lensing masses were obtained with the SL+WL method. The X-ray masses were obtained with the forward method. The discrepancies in the simulated sample are consistent with those observed in several galaxy clusters. As an example, we overplot with asterisks the mass measurements of a sample of 18 galaxy clusters reported by Mahdavi et al. (2008) (M08 in the following discussion).

Several recent studies seem to agree on the fact that the lensing masses are on average higher than the X-ray masses. For example, analyzing a sample of 19 clusters observed with SUBARU and XMM-Newton, Zhang et al. (2008) find that $M_{500, \mathrm{WL}} / M_{500, \mathrm{X}}=1.09 \pm 0.08$. M08 report similar results. In particular, they find a trend in the ratio between lensing and X-ray masses as a function of the overdensity radius. While X-ray and the lensing masses are consistent with each other at $R_{2500}$ $\left(M_{2500, \mathrm{X}} / M_{2500, \mathrm{WL}}=1.03 \pm 0.07\right)$, their mean ratio becomes $0.78 \pm 0.09$ at $R_{500}$. Correcting for excess correlated structure outside the virial radius, they find $M_{2500, \mathrm{X}} / M_{2500, \mathrm{WL}}=1.06 \pm 0.07$ and $M_{500, \mathrm{X}} / M_{500, \mathrm{wL}}=0.85 \pm 0.10$. Very recently when studying a sample of 12 clusters divided into relaxed and unrelaxed on the basis of their X-ray morphology, Zhang et al. (2010) (Z09 in the following) have also found that the weak lensing masses exceed the X-ray masses at large radii (or low overdensities), while they are comparable at small radii (or high overdensities). However, they measure a shallower but still significant radial evolution of $M_{\mathrm{X}} / M_{\mathrm{L}}$ compared to M08. For the subsample of relaxed clusters, they find $M_{2500, \mathrm{X}} / M_{2500, \mathrm{WL}}=1.04 \pm 0.08$ and $M_{500, \mathrm{X}} / M_{500, \mathrm{WL}}=0.91 \pm 0.06$ on average, while for the whole sample of objects they find $M_{2500, \mathrm{X}} / M_{2500, \mathrm{WL}}=0.97 \pm 0.07$ and $M_{500, \mathrm{X}} / M_{500, \mathrm{WL}}=0.94 \pm 0.05$. This trend is interpreted as an indication of the lack of hydrostatic equilibrium in galaxy clusters (Churazov et al. 2008).

We repeat here the analysis of M08 and Z09 using our simulated clusters. Following the terminology of M08, we indicate with $a_{\Delta}$ the ratio between the X-ray and 3D-lensing masses for a particular overdensity $\Delta$. We measure $a_{\Delta}$ for $\Delta=2500,1000$, 500, and 200. Consistently with M08 and Z09, we estimate $a_{\Delta}$ by minimizing a $\chi^{2}$ statistic defined as

$\chi^{2}=\sum \frac{\left(M_{\Delta, \mathrm{X}}-a_{\Delta} M_{\Delta, \mathrm{L}}\right)^{2}}{\sigma_{\Delta, \mathrm{X}}^{2}+a_{\Delta}^{2} \sigma_{\Delta, \mathrm{L}}^{2}}$,

where $\sigma_{\Delta, \mathrm{X}}$ and $\sigma_{\Delta, \mathrm{L}}$ are the errors on the X-ray and lensing masses corresponding to the overdensity $\Delta$. The errors on $a_{\Delta}$ are estimated by by locating the values at which $\chi^{2}-\chi_{\min }=1$, which

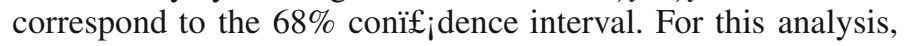
we use the lensing masses obtained from the SL+WL method and the X-ray masses obtained from both the forward and the backward methods. The lensing masses in M08 and Z09 are obtained with the NFW fit method. We opt for the SL+WL method because our sample is limited and a significant fraction of our clusters have significant substructures. Under these conditions the efficiency of the NFW fit method to obtain reliable mass estimates is limited, as we discussed in Sect. 4.1.2. The results are shown in Fig. 20, where we plot $a_{\Delta}$ as a function of $\Delta$. For comparison, we overplot the datapoints taken from Fig. 4 of M08. We also plot the results obtained by Z09 for their subsample of relaxed clusters.. We find that the ratios between X-ray and lensing 


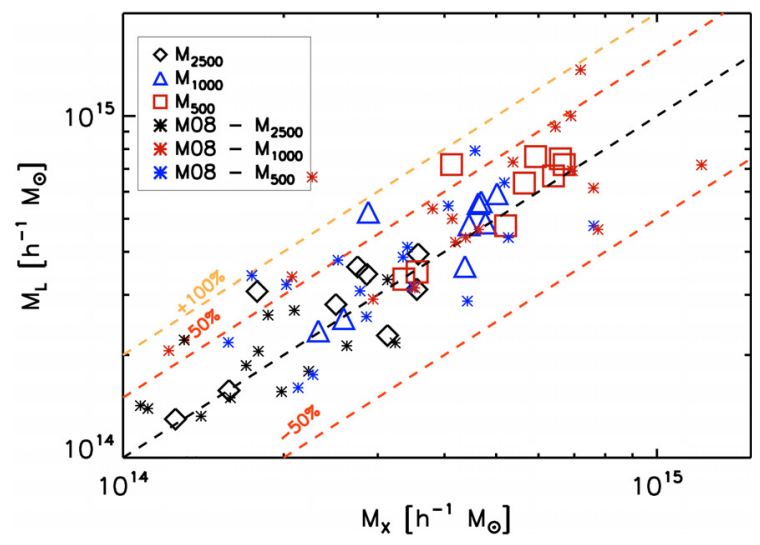

Fig. 19. Lensing vs. X-ray masses within $R_{2500}$ (diamonds), $R_{500}$ (triangles), and $R_{200}$ (squares). The asterisks show the mass estimates published by M08 for a sample of 18 galaxy clusters. The dashed lines correspond to $M_{\mathrm{L}}=[0.5,1.0,1.5,2.0] \times M_{\mathrm{X}}$.

masses are below unity at all over-density radii. For $\Delta=2500$, we find $a_{\Delta}=0.9_{-0.03}^{+0.05}$ and $a_{\Delta} \sim 0.91_{0.04}^{+0.05}$ using the masses derived from the forward and from the backward methods, respectively. For $\Delta=500$ this ratio becomes $a_{\Delta}=0.88_{-0.02}^{+0.03}$ using the forward method to measure the X-ray mass, and with the backward method it is $a_{\Delta}=0.87_{-0.04}^{+0.04}$. We do not detect a strong radial trend as reported in M08 and Z09. On the contrary, the ratios between X-ray and lensing masses decline very gently as a function of the overdensity radius.

The important question that arises now is whether the ratio between lensing and X-ray masses is a robust indicator of the lack of hydrostatic equilibrium in the simulated clusters. This can be checked easily by measuring the mass profile of the simulated clusters using the hydrostatic equilibrium approximation. For doing this, we plugged the gas density and the temperature profiles measured from the particle distributions into the hydrostatic equilibrium equation and derived the mass $M_{\mathrm{HEQ}}$. This mass should be recovered by the X-ray analysis; i.e., $M_{\mathrm{X}}$ is a measurement of $M_{\mathrm{HEQ}}$. This mass is compared to the true mass $M_{\text {true }}$ as in Rasia et al. (2004). By considering the three clusters, we obtained the median profile of the ratio between hydrostatic equilibrium and total mass, shown in Fig. 20. For comparison, we indicate the ratios between X-ray and true masses at each overdensity radius. In these simulations, the median $M_{\mathrm{HEQ}} / M_{\text {true }}$ is $\sim 0.9$ between $R_{2500}$ and $R_{1000}$, and then it decreases to 0.84 and 0.8 at $R_{500}$ and $R_{200}$. The X-ray masses are compatible (within the $68 \%$ confidence limit) with $M_{\mathrm{HEQ}}$. The mass derived with the backward method declines more steeply than what was obtained with the forward method as $\Delta$ decreases, and seems to follow the behavior of $M_{\mathrm{HEQ}}$ more closely, although the differences are not significant given the error-bars. $M_{\mathrm{X}} / M_{\mathrm{L}}$ is also compatible with $M_{\mathrm{HEQ}} / M_{\text {true }}$. This is true only at the $2 \sigma$ significance level for $\Delta<500$. Thus, we conclude that lensing and X-ray observations and their comparison to the simulations can provide important information on the physics of the gas in galaxy clusters. Although we have considered a small sample of clusters, averaging over several objects significantly reduces the impact of triaxiality. Indeed, at all overdiensities, $M_{\mathrm{X}} / M_{\mathrm{L}}$ is very similar to $M_{\mathrm{X}} / M_{\text {true }}$, indicating that $M_{\mathrm{L}} \sim M_{\text {true }}$ on average.

In Table 4 we report the mean, the rms, the median, the first and the third quartiles, and the minimal and maximal values of the distributions of several mass ratios obtained via lensing and X-ray analyses and from the input simulations. The lensing masses are measured with the SL+WL method. For the X-ray

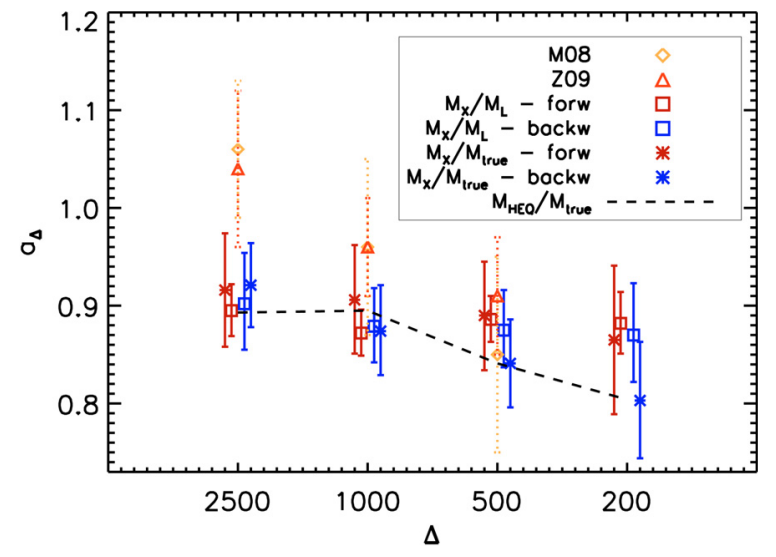

Fig. 20. Ratio between $X$-ray and lensing masses as a function of the overdensity $\Delta$ (squares). The results are shown for the X-ray masses obtained with the forward (red) and with the backward (blue) methods and for the lensing masses obtained with the SL+WL method. For comparison, we also show the ratios between the X-ray masses and the true masses of the clusters (asterisks) and the ratios between the masses determined via the hydrostatic equilibrium equation using the true gas density and temperature profiles and the true masses (dashed line). The diamonds and the triangles show the results published by M08 and Z09, based on the analysis of a sample of 18 and 12 galaxy clusters, respectively. The datapoints are slightly shifted along the $\Delta$-axis at each overdensity, in order to avoid overlapping and facilitating the comparisons.

masses we use both the forward and the backward methods. Not only do we compare 3D vs. 3D masses, but we also compare $3 \mathrm{D}$ vs. $2 \mathrm{D}$ mass estimates and vice-versa. We note that triaxiality and substructure affect the 2D X-ray mass estimates consistently to the 3D lensing mass estimates. For example the $\mathrm{rms}$ of the ratio $M_{2 \mathrm{D}, \mathrm{X}} / M_{2 \mathrm{D}, \mathrm{L}}$ is $\sim 17-18 \%$, similar to the rms of the ratio $M_{3 \mathrm{D}, \mathrm{X}} / M_{3 \mathrm{D}, \mathrm{L}}$. Not surprisingly, the mass ratio that has the smaller scatter is $M_{3 \mathrm{D}, \mathrm{X}} / M_{2 \mathrm{D}, \mathrm{L}}$, whose $\mathrm{rms}$ is $\sim 13 \%$. However, the radial dependence of this mass ratio is stronger than for $M_{3 \mathrm{D}, \mathrm{X}} / M_{3 \mathrm{D}, \mathrm{L}}$ or $M_{2 \mathrm{D}, \mathrm{X}} / M_{2 \mathrm{D}, \mathrm{L}}$ as indicated by the variation of the mean and of the median as a function of the overdensity $\Delta$. Obviously, the mass ratio affected by the largest scatter is $M_{2 \mathrm{D}, \mathrm{X}} / M_{3 \mathrm{D}, \mathrm{L}}(\sim 25 \%)$.

\section{Summary and conclusions}

In this paper, we have tested several methods of deriving the mass of galaxy clusters via lensing and X-ray observations. To do so, we used two pipelines for simulating observations of galaxy clusters obtained from $N$-body and hydrodynamical simulations. These are the code SkyLens, which produces mock observations with a variety of telescopes in the optical bands, including lensing effects by matter along the line of sight, and the code XMAS, which allows mimicking of X-ray observations of galaxy clusters with the Chandra and the XMM-Newton telescopes. In our analysis, we used three massive galaxy clusters at redshifts between $z=0.2335$ and $z=0.297$. Each cluster was projected along three orthogonal axes and each projection was analyzed individually. For each cluster projection, we carried out both the lensing and the X-ray analysis using standard techniques to derive the mass profiles.

Our results can be summarized as follows.

- Strong lensing parametric mass reconstructions provide very accurate estimates of the projected mass, but only in the regions probed by the strong lensing features. Our simulations show that, within the Einstein rings, the input masses are 
A\&A 514, A93 (2010)

Table 4. Summary of the comparison between lensing, X-ray, and true masses.

\begin{tabular}{|c|c|c|c|c|c|c|c|c|}
\hline Ratio & $\Delta$ & Mean & $\mathrm{rms}$ & Median & 1st quart. & 3rd quart. & Min & Max \\
\hline$M_{3 \mathrm{D} X \mathrm{X}}^{\text {forw }} / M_{3 \mathrm{D} \text {,true }}$ & 2500 & 0.9241 & 0.1147 & 0.8854 & 0.8451 & 0.9730 & 0.8007 & 1.1143 \\
\hline$M_{3 \mathrm{D} \text { X }}^{\text {forw }} / M_{3 \mathrm{D} \text {,true }}$ & 500 & 0.8811 & 0.0538 & 0.8950 & 0.8594 & 0.9059 & 0.7640 & 0.9493 \\
\hline$M_{3 \mathrm{D}, \mathrm{X}}^{\text {forw }} / M_{3 \mathrm{D} \text {, true }}$ & 200 & 0.8947 & 0.0625 & 0.9162 & 0.8534 & 0.9293 & 0.7749 & 0.9759 \\
\hline$M_{3 \mathrm{D} \mathrm{N}}^{\text {back }} / M_{3 \mathrm{D} \text {,true }}$ & 2500 & 0.9205 & 0.0690 & 0.9018 & 0.8669 & 0.9669 & 0.8512 & 1.0407 \\
\hline$M_{3 \mathrm{D}, \mathrm{X}}^{\text {back }} / M_{3 \mathrm{D} \text {,true }}$ & 500 & 0.8961 & 0.0582 & 0.8826 & 0.8817 & 0.9288 & 0.7753 & 0.9853 \\
\hline$M_{3 \mathrm{D}, \mathrm{X}}^{\text {back }} / M_{3 \mathrm{D} \text {,true }}$ & 200 & 0.8832 & 0.0664 & 0.9019 & 0.8878 & 0.9126 & 0.7192 & 0.9559 \\
\hline$M_{3 \mathrm{D}, \mathrm{L}} / M_{3 \mathrm{D} \text {,true }}$ & 2500 & 1.0233 & 0.1686 & 0.9683 & 0.9093 & 1.1033 & 0.8290 & 1.3687 \\
\hline$M_{3 \mathrm{D}, \mathrm{L}} / M_{3 \mathrm{D}, \text { true }}$ & 500 & 0.9963 & 0.1661 & 0.9374 & 0.8930 & 1.0251 & 0.8362 & 1.3438 \\
\hline$M_{3 \mathrm{D}, \mathrm{L}} / M_{3 \mathrm{D} \text {,true }}$ & 200 & 0.9807 & 0.1734 & 0.8891 & 0.8682 & 1.0783 & 0.8234 & 1.2879 \\
\hline$M_{3 \mathrm{D}, \mathrm{X}}^{\text {forw }} / M_{3 \mathrm{D}, \mathrm{L}}$ & 2500 & 0.9303 & 0.2193 & 0.8917 & 0.8303 & 1.0034 & 0.5850 & 1.3441 \\
\hline$M_{3 \mathrm{D}, \mathrm{X}}^{\mathrm{for},} / M_{3 \mathrm{D}, \mathrm{L}}$ & 500 & 0.9062 & 0.1521 & 0.9429 & 0.8837 & 1.0167 & 0.5686 & 1.0768 \\
\hline$M_{3 \mathrm{D}, \mathrm{X}}^{\mathrm{fD}, \mathrm{d}} / M_{3 \mathrm{D}, \mathrm{L}}$ & 200 & 0.9395 & 0.1802 & 1.0091 & 0.7915 & 1.0815 & 0.6017 & 1.1127 \\
\hline$M_{3 \mathrm{D} X}^{\text {back }} / M_{3 \mathrm{D}, \mathrm{L}}$ & 2500 & 0.9230 & 0.1776 & 0.9109 & 0.8416 & 0.9934 & 0.6219 & 1.2553 \\
\hline$M_{3 \mathrm{DX}}^{\text {back }} / M_{3 \mathrm{D}, \mathrm{L}}$ & 500 & 0.9244 & 0.1731 & 0.9415 & 0.8926 & 1.0690 & 0.5770 & 1.1108 \\
\hline$M_{3 \mathrm{D}, \mathrm{X}}^{\mathrm{back}} / M_{3 \mathrm{D}, \mathrm{L}}$ & 200 & 0.9307 & 0.1937 & 1.0088 & 0.8374 & 1.0541 & 0.5584 & 1.1353 \\
\hline$M_{2 \mathrm{D}, \mathrm{L}} / M_{3 \mathrm{D} \text {,true }}$ & 2500 & 1.4898 & 0.2485 & 1.4317 & 1.3671 & 1.5457 & 1.1611 & 2.0612 \\
\hline$M_{2 \mathrm{D}, \mathrm{L}} / M_{3 \mathrm{D}, \text { true }}$ & 500 & 1.2924 & 0.2151 & 1.2127 & 838 & 3059 & 1.0607 & 1.7788 \\
\hline$M_{2 \mathrm{D}, \mathrm{L}} / M_{3 \mathrm{D} \text {,true }}$ & 200 & 1.2171 & 0.2092 & 1.1109 & 1.0700 & 1.3212 & 1.0545 & 1.6244 \\
\hline$M_{2 \mathrm{DX}}^{\text {forw }} / M_{3 \mathrm{D} \text {,true }}$ & 2500 & 1.2850 & 0.0757 & 1.2744 & 1.2627 & 1.3288 & 1.1276 & 1.3839 \\
\hline$M_{2 \mathrm{D} \mathrm{X}}^{\text {for, }} / M_{3 \mathrm{D} \text {,true }}$ & 500 & 1.0477 & 0.0852 & 1.0376 & 0.9853 & 1.0810 & 0.9179 & 1.1760 \\
\hline$M_{2 \mathrm{D}, \mathrm{X}}^{\text {forw }} / M_{3 \mathrm{D} \text {,true }}$ & 200 & 0.8961 & 0.0835 & 0.8839 & 0.8446 & 0.9382 & 0.7449 & 1.0162 \\
\hline$M_{2 \mathrm{D}, \mathrm{X}}^{\text {back }} / M_{3 \mathrm{D} \text {,true }}$ & 2500 & 1.3211 & 0.0754 & 1.3317 & 1.2903 & 1.3520 & 1.2052 & 1.4415 \\
\hline$M_{2 \mathrm{D}, \mathrm{X}}^{\text {back }} / M_{3 \mathrm{D} \text {,true }}$ & 500 & 1.1164 & 0.1121 & 1.1106 & 1.0643 & 1.1270 & 0.9497 & 1.3130 \\
\hline$M_{2 \mathrm{D}, \mathrm{X}}^{\text {back }} / M_{3 \mathrm{D} \text {,rue }}$ & 200 & 1.0124 & 0.0990 & 1.0200 & 0.9919 & 1.0436 & 0.8108 & 1.1585 \\
\hline$M_{2 \mathrm{D}, \mathrm{X}}^{\mathrm{forw}} / M_{2 \mathrm{D}, \mathrm{L}}$ & 2500 & 0.8875 & 0.1733 & 0.8901 & 0.8221 & 0.9779 & 0.5471 & 1.1702 \\
\hline$M_{2 \mathrm{D}, \mathrm{X}}^{\mathrm{for}, \mathrm{d}} / M_{2 \mathrm{D}, \mathrm{L}}$ & 500 & 0.8314 & 0.1503 & 0.8790 & 0.7545 & 0.9272 & 0.5160 & 0.9834 \\
\hline$M_{2 \mathrm{D}, \mathrm{X}}^{\mathrm{for}} / M_{2 \mathrm{D}, \mathrm{L}}$ & 200 & 0.7596 & 0.1629 & 0.7949 & 0.6388 & 0.8664 & 0.4586 & 0.9636 \\
\hline$M_{2 \mathrm{D} X}^{\text {back }} / M_{2 \mathrm{D}, \mathrm{L}}$ & 2500 & 0.9103 & 0.1657 & 0.9301 & 0.8634 & 1.0259 & 0.5847 & 1.1584 \\
\hline$M_{2 \mathrm{D}, \mathrm{X}}^{\text {back }} / M_{2 \mathrm{D}, \mathrm{L}}$ & 500 & & 0.1825 & 0.9475 & 0.8150 & 1.0299 & 0.5339 & 1.0827 \\
\hline$M_{2 \mathrm{D}, \mathrm{X}}^{\mathrm{back}} / M_{2 \mathrm{D}, \mathrm{L}}$ & 200 & 0.8609 & 0.1951 & 0.9259 & 0.7507 & 0.9638 & 0.4991 & 1.0827 \\
\hline$M_{2 \mathrm{DX}}^{\text {forw }} / M_{3 \mathrm{D}, \mathrm{L}}$ & 2500 & 1.2929 & 0.2579 & 1.3392 & 1.1445 & 1.5218 & 0.8239 & 1.6389 \\
\hline$M_{2 \mathrm{D} X}^{\mathrm{for},} / M_{3 \mathrm{D}, \mathrm{L}}$ & 500 & 1.0801 & 0.2064 & 1.1532 & 0.9612 & 1.1762 & 0.6830 & 1.3297 \\
\hline$M_{2 \mathrm{D}, \mathrm{X}}^{\text {forw }} / M_{3 \mathrm{D}, \mathrm{L}}$ & 200 & 0.9450 & 0.2123 & 0.9728 & 0.7828 & 1.0552 & 0.5783 & 1.2341 \\
\hline$M_{2 \mathrm{D} X}^{\text {back }} / M_{3 \mathrm{D}, \mathrm{L}}$ & 2500 & 1.3274 & 0.2560 & 1.3326 & 1.2020 & 1.5853 & 0.8806 & 1.6223 \\
\hline$M_{2 \mathrm{DX}}^{\text {back }} / M_{3 \mathrm{D}, \mathrm{L}}$ & 500 & 1.1553 & 0.2502 & 1.2023 & 1.0383 & 1.3064 & 0.7067 & 1.4481 \\
\hline$M_{2 \mathrm{D}, \mathrm{X}}^{\text {back }} / M_{3 \mathrm{D}, \mathrm{L}}$ & 200 & 1.0711 & 0.2524 & 1.1663 & 0.9198 & 1.1748 & 0.6295 & 1.3760 \\
\hline$M_{3 \mathrm{DX}}^{\text {forw }} / M_{2 \mathrm{D}, \mathrm{L}}$ & 2500 & 0.6407 & 0.1612 & 0.6212 & 0.5728 & 0.6556 & 0.3885 & 0.9597 \\
\hline$M_{3 \mathrm{DX}}^{\mathrm{forw}} / M_{2 \mathrm{D}, \mathrm{L}}$ & 500 & 0.6985 & 0.1166 & 0.7281 & 0.6838 & 0.7519 & 0.4295 & 0.8489 \\
\hline$M_{3 \mathrm{D}, \mathrm{X}}^{\mathrm{for},} / M_{2 \mathrm{D}, \mathrm{L}}$ & 200 & 0.7557 & 0.1407 & 0.8147 & 0.6459 & 0.8509 & 0.4771 & 0.9013 \\
\hline$M_{3 \mathrm{D} X}^{\text {back }} / M_{2 \mathrm{D}, \mathrm{L}}$ & 2500 & 0.6352 & 0.1302 & 0.6173 & 0.5895 & 0.6479 & 0.4129 & 0.8963 \\
\hline$M_{3 \mathrm{D} X}^{\text {back }} / M_{2 \mathrm{D}, \mathrm{L}}$ & 500 & 0.7120 & 0.1299 & 0.7391 & 0.7006 & 0.7906 & 0.4359 & 0.8757 \\
\hline$M_{3 \mathrm{D}, \mathrm{X}}^{\text {back }} / M_{2 \mathrm{D}, \mathrm{L}}$ & 200 & 0.7485 & 0.1510 & 0.8009 & 0.6834 & 0.8633 & 0.4428 & 0.8933 \\
\hline
\end{tabular}

recovered with an accuracy of $\$ 20 \%$. For most cases, the measured masses differ from the input ones by only a few percent. The precision depends on the complexity of the lens and on the number of lensing constraints available. For example, using central images improves the mass estimates in the very inner regions. On the other hand, extrapolating the mass models to radii larger than those probed by strong lensing may easily result in mass estimates that are wrong by up to $40-50 \%$. In particular, the assumptions made for modeling the star distribution in the BCG affect the mass profiles at large radii. Although the very central regions of numerically simulated clusters may be not representative of those of real clusters, given the uncertainties on the treatment of small-scale physics of the intracluster gas, our results warn against potential biases that may affect the strong lensing mass estimates.

- Weak lensing methods for measuring the projected mass perform well with galaxy clusters characterized by regular mass distributions, i.e. without massive substructures that produce a significant shear. If present, such substructures need to be properly modeled. If not, the cluster masses can be over- or under-estimated by up to $50 \%$, when assuming that the shear signal is tangential to a unique mass center. Nonparametric methods combining weak and strong lensing, which by construction do not assume any symmetry in the lensing signal, perform well even in the case of clusters disturbed by 
massive substructures. The mass estimates at $R_{2500}, R_{500}$, and $R_{200}$ are typically recovered within a $20 \%$ accuracy, being more accurate at the smallest radii.

- Deprojection of the lensing masses introduces a significant scatter in the ratio between estimated and true masses. Cluster triaxiality causes that the accuracy of 3D-mass estimates depends on the orientation of the lens with respect to the line of sight. For clusters whose major axis points towards the observer the $3 \mathrm{D}$-mass is over-estimated, while the opposite happens for clusters oriented perpedicularly to the line of sight. Cluster substructures also cause errors during the deprojection, as the distance of the substructures from the cluster center along the line of sight is unknown.

- X-ray masses are typically biased low by $5-20 \%$ due to the lack of hydrostatic equilibrium in the simulated clusters. The average bias between $R_{2500}$ and $R_{200}$ is $\sim 10 \%$. In fact, the accretion of material from the surroundings during the cluster formation process causes bulk motions that contribute to the pressure support of the gas. As shown in previous works, this effect is radial dependent, i.e. it is stronger in the outer regions. We investigated whether the ratio of X-ray-to-lensing masses $a_{\Delta}$ can be used as a tracer of hydrostatic equilibrium. By averaging over the sample, we find that this ratio indeed reproduces the lack of hydrostatic equilibrium in the input simulations, although the number of available clusters is limited and despite the effects of triaxiality and substructures which affect the cluster 3D-mass estimates.

- The gas mass is reconstructed well within the region from which we can extract a surface brightness profile. Almost indipendently of the dynamical state of the cluster, the gas mass can be recovered, on average, with an average deviation of $1 \pm 3 \%$ at $R_{2500}$ and $7 \pm 4 \%$ between $R_{500}$ and $R_{200}$.

These results indicate that the comparison between lensing and $\mathrm{X}$-ray mass estimates in individual systems can be very misleading, because these two observables probe different quantities. While lensing probes the matter projected along the line of sight to the clusters, the X-ray emission from the intracluster gas probes the 3D gravitational potential. Thus, a direct comparison between lensing and X-ray masses is possible only in those systems which are characterized by high degrees of spherical symmetry. Additional complications are caused by substructures, which largely affect the weak lensing measurements, at least using some methods of converting the shear into a mass estimate. Combining strong and weak lensing, we obtained on average a very small bias in the mass estimates $(\sim 2 \%)$, but the scatter is fairly high $(\sim 17-23 \%)$. In contrast, X-ray masses are affected by a more substantial bias $(\sim 10 \%)$, but the scatter is smaller by almost a factor of three.

Finally, strong lensing alone provides mass estimates that are reliable only in a very small region around the cluster core, which cannot be probed by X-ray. Extrapolating the strong lensing mass models to the radii probed by X-ray (and vice-versa) may result in significant mismatches between mass estimates. Note, however, that the difficulty of fitting the strong lensing data in simulations with cooling and star formation with the approximations that are commonly used in observations is very likely caused by the unrealistic core structure of the simulated clusters.

Nevertheless, it is remarkable that, by averaging over the whole sample, we have been able to reproduce the ratio between true and hydrostatic equilibrium masses seen in the simulations, under the assumption that the former is given by lensing and the latter by X-ray. This suggests that, with a sufficiently large sample of clusters with wide-field imaging and X-ray observations, the impact of several noises affecting the mass estimates can be reduced. This is positive news that suggests that weak lensing masses may be used as reliable calibrators for the scaling relations involving the $\mathrm{X}$-ray observables, such as the $M-L_{\mathrm{X}}$, the $M-T_{\mathrm{X}}$, and the $M-Y_{\mathrm{X}}$ relations. Moreover, the scatter due to triaxiality in the calibration can potentially be reduced. In fact, although its radial dependence is stronger, the ratio between 3D X-ray masses and 2D lensing masses is affected by a smaller scatter than the ratio between $3 \mathrm{D}$ or $2 \mathrm{D}$ X-ray and lensing masses.

Acknowledgements. We acknowledge financial contribution from contracts ASI-INAF I/023/05/0, ASI-INAF I/088/06/0, and INFN PD51. Support for this work was also provided by NASA through Chandra Postdoctoral Fellowship grant number PF5-70042 awarded by the Chandra X-ray Center, which is operated by the Smithsonian Astrophysical Observatory for NASA under the contract NAS8-03060. E.R. acknoweldges the Michigan Society of Fellows. We are grateful to L. Moscardini for having carefully read the manuscript and provided useful comments. M.M. thanks Yu-Ying Zhang and collaborators for providing a draft of their paper. M.M. thanks the Institut für Theoretische Astrophysik of the University of Heidelberg for hospitality during the preparation of this manuscript. The work was performed under the HPC-EUROPA2 project (project number: 228398) with the support of the European Commission - Capacities Area - Research Infrastructures. We are grateful to Y. Suto and S. Miyazaki for their help implementing the Subaru interface in the SkyLens simulator. M.M. thanks Paolo for his cooperation during the preparation of the manuscript.

\section{Appendix A: Lensing definitions}

In this section, we summarize some lensing definitions that will be useful in the rest of the paper.

We start with an isolated lens whose surface-mass density is $\Sigma(\boldsymbol{\theta})$ at the angular position $\boldsymbol{\theta}$ on the sky. Its lensing potential is

$\psi(\boldsymbol{\theta})=\frac{4 G}{c^{2}} \frac{D_{1} D_{\mathrm{s}}}{D_{\mathrm{ls}}} \int \mathrm{d}^{2} \theta^{\prime} \Sigma\left(\boldsymbol{\theta}^{\prime}\right) \ln \left|\boldsymbol{\theta}-\boldsymbol{\theta}^{\prime}\right|$,

where $D_{1, \mathrm{~s}, \mathrm{ls}}$ are the usual angular-diameter distances between the observer and the lens, the observer and the source, and the lens and the source, respectively. The reduced deflection angle experienced by a light ray crossing the lens plane at $\boldsymbol{\theta}$ is the gradient of the potential,

$\alpha(\theta)=\nabla \psi(\theta)$.

The image positions $\boldsymbol{\theta}$ for a source located at $\boldsymbol{\beta}$ are given by the lens equation

$\beta=\theta-\alpha(\theta)$.

For sources much smaller than the typical scales on which the lens properties vary, the lens mapping can be linearized. The deformation of images with respect to the source is then given by the Jacobian matrix

$\mathcal{A} \equiv \frac{\partial \boldsymbol{\beta}}{\partial \boldsymbol{\theta}}=\left(\delta_{i j}-\frac{\partial^{2} \psi(\boldsymbol{\theta})}{\partial \theta_{i} \partial \theta_{j}}\right)=\left(\begin{array}{cc}1-\kappa-\gamma_{1} & -\gamma_{2} \\ -\gamma_{2} & 1-\kappa+\gamma_{1}\end{array}\right)$.

Here, $\kappa$ is the convergence

$\kappa(\boldsymbol{\theta})=\frac{\Sigma(\boldsymbol{\theta})}{\Sigma_{\mathrm{cr}}}=\frac{1}{2}\left(\psi_{11}+\psi_{22}\right)$,

i.e. the surface-mass density scaled by its critical value

$\Sigma_{\mathrm{cr}}=\frac{c^{2}}{4 \pi G} \frac{D_{\mathrm{ls}}}{D_{\mathrm{l}} D_{\mathrm{s}}}$

The distortion is described by the two components of the shear,

$\gamma_{1}=\frac{1}{2}\left(\psi_{11}-\psi_{22}\right), \quad \gamma_{2}=\psi_{12}$ 

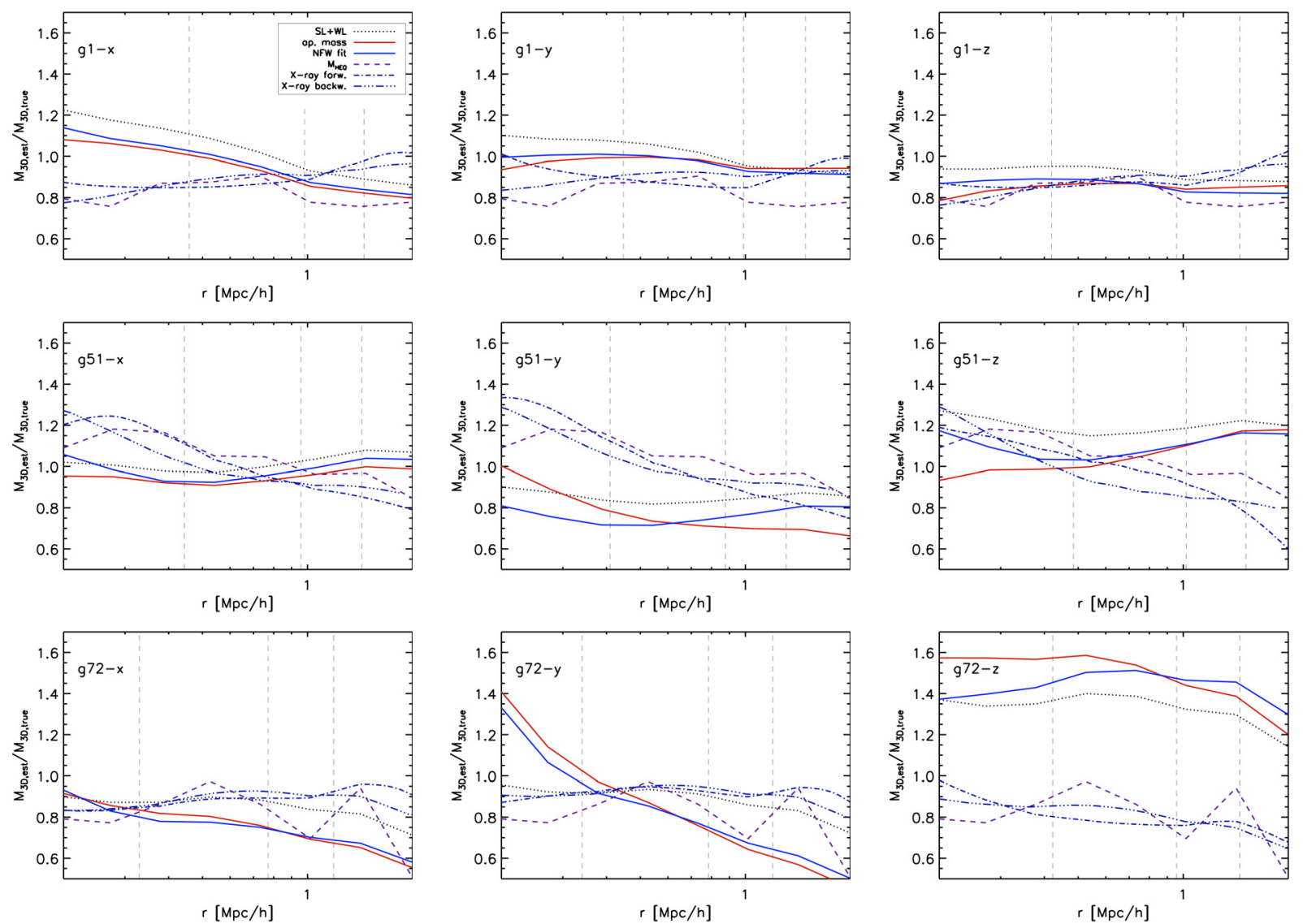

Fig. B.1. Ratios between the mass profiles recovered from the lensing and the X-ray analyses and the true 3D mass profiles of each cluster. The meaning of each line is explained in the legend. The vertical dashed lines in each panel mark the positions of $R_{2500}, R_{500}$, and $R_{200}$, as derived from the lensing analysis (see text for more details).

which are combined into the complex shear $\gamma \equiv \gamma_{1}+\mathrm{i} \gamma_{2}$. We then use the common abbreviation

$$
\frac{\partial^{2} \psi(\boldsymbol{\theta})}{\partial \theta_{i} \partial \theta_{j}} \equiv \psi_{i j}
$$

The inverse of the Jacobian determinant defines the magnification factor

$\mu(\boldsymbol{\theta})=\frac{1}{\operatorname{det} \mathcal{A}(\boldsymbol{\theta})}$.

The loci on the image plane where $\operatorname{det} \mathcal{A}=0$, i.e. where the magnification diverges, are the tangential and the radial critical lines. These form where the tangential and the radial eigenvalues of the Jacobian determinant nullifies:

$\lambda_{\mathrm{t}}=1-\kappa-\gamma=0$
$\lambda_{\mathrm{r}}=1-\kappa+\gamma=0$.

The inverses of the eigenvalues of the Jacobian matrix define the tangential and the radial magnifications: images forming close to the tangential or to the radial critical lines are highly elongated tangentially or perpendicularly to the critical lines, respectively. The critical lines are mapped onto the caustics on the source plane, via the lens equation. The caustics encompass regions of the source plane, which are characterized by different multiplicities of the images.

Outside critical curves, image ellipticities are determined by the complex reduced shear

$g(\boldsymbol{\theta}) \equiv \frac{\gamma(\boldsymbol{\theta})}{1-\kappa(\boldsymbol{\theta})}$
In the weak-lensing limit, $\kappa \ll 1$, and the reduced shear approximates the shear, $g \approx \gamma$, to first order.

Source and image shapes are quantified by the complex ellipticity

$\epsilon=\frac{a-b}{a+b} \mathrm{e}^{2 \mathrm{i} \vartheta}$,

where $a$ and $b$ are the semi-major and semi-minor axes of an ellipse fitting the object's surface-brightness distribution. The position angle of the ellipse's major axis is $\vartheta$. The expected value of the intrinsic source ellipticity $\epsilon_{\mathrm{s}}$ is assumed to vanish.

A sufficiently small source with ellipticity $\epsilon_{\mathrm{s}}$ is imaged to have an ellipticity

$\epsilon=\frac{\epsilon_{\mathrm{s}}+g}{1+g^{*} \epsilon_{\mathrm{s}}}$,

where the asterisk denotes complex conjugation. This equation illustrates that the lensing distortion is determined by the reduced shear, which is the only lensing quantity directly accessible through measurements of galaxy ellipticities alone.

\section{Appendix B: Individual mass profiles}

In this section we show the 3D-mass profiles (normalized to the true mass profiles) of each individual cluster projection in our sample. This allows the reader to compare the mass estimates at any radius different from $R_{2500}, R_{500}$, and $R_{200}$, which are already discussed in the paper. The profiles are displayed in Fig. B.1 for all the lensing and the X-ray methods. Additionally, we also 
show the mass profiles derived from the true gas and temperature profiles under the assumption of hydrostatic equilibrium. These last curves are quite noisy, especially in the case of $g 72$, because we did not apply any smoothing to the data. In the case of $g 51$, the extended cooling region in the cluster center causes the hydrostatic mass $M_{\mathrm{HEQ}}$ to be higher than the true mass. The $\mathrm{X}$-ray derived profiles reproduce the trends seen in the $M_{\mathrm{HEQ}}$ profiles although some differences are present. While we mask many cold blobs in the simulated observations, we are not removing these structures from the input clusters when calculating $M_{\mathrm{HEQ}}$. Thus, some differences between the profiles are expected.

\section{References}

Allen, S. 1998, MNRAS, 296, 392

Allen, S. W., Ettori, S., \& Fabian, A. C. 2001, MNRAS, 324, 877

Allen, S. W., Rapetti, D. A., Schmidt, R. W., et al. 2008, MNRAS, 383, 879

Allen, S. W., Schmidt, R. W., Ebeling, H., Fabian, A. C., \& van Speybroeck, L. 2004, MNRAS, 353, 457

Ameglio, S., Borgani, S., Pierpaoli, E., et al. 2009, MNRAS, 394, 479

Arnaud, K. A. 1996, in Astronomical Data Analysis Software and Systems V, ed. G. H. Jacoby, \& J. Barnes, ASP Conf. Ser., 101, 17

Aubert, D., Amara, A., \& Metcalf, R. B. 2007, MNRAS, 376, 113

Bartelmann, M. 1995, A\&A, 299, 11

Bartelmann, M. 1996, A\&A, 313, 697

Bartelmann, M., \& Schneider, P. 2001, Phys. Rep., 340, 291

Bartelmann, M., \& Steinmetz, M. 1996, MNRAS, 283, 431

Beckwith, S. V. W., Stiavelli, M., Koekemoer, A. M., et al. 2006, AJ, 132, 1729

Benítez, N. 2000, ApJ, 536, 571

Bradač, M., Erben, T., Schneider, P., et al. 2005, A\&A, 437, 49

Buote, D. A., Gastaldello, F., Humphrey, P. J., et al. 2007, ApJ, 664, 123

Cen, R. 1997, ApJ, 485, 39

Churazov, E., Forman, W., Vikhlinin, A., et al. 2008, MNRAS, 388, 1062

Clowe, D., Gonzalez, A., \& Markevitch, M. 2004, ApJ, 604, 596

Clowe, D., Luppino, G. A., Kaiser, N., Henry, J. P., \& Gioia, I. M. 1998, ApJ, 497, L61

Coe, D., Benítez, N., Sánchez, S. F., et al. 2006, AJ, 132, 926

Comerford, J. M., Meneghetti, M., Bartelmann, M., \& Schirmer, M. 2006, ApJ, 642,39

Comerford, J. M., \& Natarajan, P. 2007, MNRAS, 379, 190

Corless, V. L., \& King, L. J. 2007, MNRAS, 380, 149

Dolag, K., Vazza, F., Brunetti, G., \& Tormen, G. 2005, MNRAS, 364, 753

Donnarumma, A., Ettori, S., Meneghetti, M., \& Moscardini, L. 2009, MNRAS, 398,438

Eisenstein, D. J., Zehavi, I., Hogg, D. W., et al. 2005, ApJ, 633, 560

Ettori, S., \& Lombardi, M. 2003, A\&A, 398, L5

Ettori, S., De Grandi, S., \& Molendi, S. 2002, A\&A, 391, 841

Ettori, S., Tozzi, P., \& Rosati, P. 2003, A\&A, 398, 879

Evrard, A. E., Metzler, C. A., \& Navarro, J. F. 1996, ApJ, 469, 494

Fahlman, G., Kaiser, N., Squires, G., \& Woods, D. 1994, ApJ, 437, 56

Gardini, A., Rasia, E., Mazzotta, P., et al. 2004, MNRAS, 351, 505

Gavazzi, R. 2005, A\&A, 443, 793

Giavalisco, M., Ferguson, H. C., Koekemoer, A. M., et al. 2004, ApJ, 600, L93

Golse, G., Kneib, J.-P., \& Soucail, G. 2002, A\&A, 387, 788

Henriksen, M. J., \& Mushotzky, R. F. 1986, ApJ, 302, 287

Hoekstra, H. 2001, A\&A, 370, 743

Hoekstra, H. 2003, MNRAS, 339, 1155

Hoekstra, H. 2007, MNRAS, 379, 317

Hoekstra, H., Franx, M., Kuijken, K., \& Squires, G. 1998, ApJ, 504, 636

Jenkins, A., Frenk, C., White, S., et al. 2001, MNRAS, 321, 372

Jullo, E., Kneib, J.-P., Limousin, M., et al. 2007, New J. Phys., 9, 447

Kaiser, N., Squires, G., \& Broadhurst, T. 1995, ApJ, 449, 460

Kneib, J., Mellier, Y., Fort, B., \& Mathez, G. 1993, A\&A, 273, 367

Komatsu, E., Dunkley, J., Nolta, M. R., et al. 2009, ApJS, 180, 330

Kravtsov, A. V., Vikhlinin, A., \& Nagai, D. 2006, ApJ, 650, 128
Lau, E. T., Kravtsov, A. V., \& Nagai, D. 2009, ApJ, 705, 1129

Liedahl, D. A., Osterheld, A. L., \& Goldstein, W. H. 1995, ApJ, 438, L115

Limousin, M., Richard, J., Jullo, E., et al. 2007, ApJ, 668, 643

Luppino, G. A., \& Kaiser, N. 1997, ApJ, 475, 20

Mahdavi, A., Hoekstra, H., Babul, A., \& Henry, J. P. 2008, MNRAS, 384, 1567

Mantz, A., Allen, S. W., \& Rapetti, D. 2009a, MNRAS, accepted [arXiv:0911.1788]

Mantz, A., Allen, S. W., Rapetti, D., \& Ebeling, H. 2009b, MNRAS, submitted [arXiv:0909.3098]

Markevitch, M., Gonzalez, A. H., Clowe, D., et al. 2004, ApJ, 606, 819

Maturi, M., Meneghetti, M., Bartelmann, M., Dolag, K., \& Moscardini, L. 2005, A\&A, 442, 851

Medezinski, E., Broadhurst, T., Umetsu, K., et al. 2007, ApJ, 663, 717

Meneghetti, M., Argazzi, R., Pace, F., et al. 2007, A\&A, 461, 25

Meneghetti, M., Bartelmann, M., \& Moscardini, L. 2003a, MNRAS, 346, 67

Meneghetti, M., Bartelmann, M., \& Moscardini, L. 2003b, MNRAS, 340, 105

Meneghetti, M., Melchior, P., Grazian, A., et al. 2008, A\&A, 482, 403

Merten, J., Cacciato, M., Meneghetti, M., Mignone, C., \& Bartelmann, M. 2009, A\&A, 500, 681

Metzler, C. A., White, M., Norman, M., \& Loken, C. 1999, ApJ, 520, L9

Mewe, R., Gronenschild, E. H. B. M., \& van den Oord, G. H. J. 1985, A\&AS, 62, 197

Nagai, D., Vikhlinin, A., \& Kravtsov, A. V. 2007, ApJ, 655, 98

Navarro, J., Frenk, C., \& White, S. 1997, ApJ, 490, 493

Oguri, M., Takada, M., Umetsu, K., \& Broadhurst, T. 2005, ApJ, 632, 841

Okabe, N., Takada, M., Umetsu, K., Futamase, T., \& Smith, G. P. 2009, [arXiv:0903.1103]

Ota, N., Pointecouteau, E., Hattori, M., \& Mitsuda, K. 2004, ApJ, 601, 120

Percival, W. J., Cole, S., Eisenstein, D. J., et al. 2007, MNRAS, 381, 1053

Perlmutter, S., Aldering, G., Goldhaber, G., Knop, R., et al. 1999, ApJ, 517, 565

Piffaretti, R., \& Valdarnini, R. 2008, A\&A, 491, 71

Pointecouteau, E., Arnaud, M., \& Pratt, G. W. 2005, A\&A, 435, 1

Press, W., \& Schechter, P. 1974, ApJ, 187, 425

Puchwein, E., Bartelmann, M., Dolag, K., \& Meneghetti, M. 2005, A\&A, 442, 405

Rasia, E., Ettori, S., Moscardini, L., et al. 2006, MNRAS, 369, 2013

Rasia, E., Mazzotta, P., Bourdin, H., et al. 2008, ApJ, 674, 728

Rasia, E., Tormen, G., \& Moscardini, L. 2004, MNRAS, 351, 237

Reblinsky, K., \& Bartelmann, M. 1999, A\&A, 345, 1

Refregier, A. 2003, MNRAS, 338, 35

Riemer-Sørensen, S., Paraficz, D., Ferreira, D. D. M., et al. 2009, ApJ, 693, 1570

Riess, A., Strolger, L.-G., Tonry, J., et al. 2004, ApJ, 607, 665

Riess, A. G., Filippenko, A. V., Challis, P., et al. 1998, AJ, 116, 1009

Salpeter, E. E. 1955, ApJ, 121, 161

Sand, D. J., Treu, T., Ellis, R. S., Smith, G. P., \& Kneib, J.-P. 2008, ApJ, 674, 711

Sarazin, C. L. 1988, X-ray emission from clusters of galaxies, ed. C. L. Sarazin

Saro, A., Borgani, S., Tornatore, L., et al. 2006, MNRAS, 373, 397

Schmidt, R. W., \& Allen, S. W. 2007, MNRAS, 379, 209

Sheth, R., \& Tormen, G. 2002, MNRAS, 329, 61

Smith, G. P., Kneib, J.-P., Smail, I., et al. 2005, MNRAS, 359, 417

Springel, V. 2005, MNRAS, 364, 1105

Springel, V., White, S. D. M., Tormen, G., \& Kauffmann, G. 2001, MNRAS, 328,726

Tormen, G., Bouchet, F., \& White, S. 1997, MNRAS, 286, 865

Tornatore, L., Borgani, S., Matteucci, F., Recchi, S., \& Tozzi, P. 2004, MNRAS, 349, L19

Tornatore, L., Borgani, S., Dolag, K., \& Matteucci, F. 2007, MNRAS, 382, 1050 Vikhlinin, A., Kravtsov, A. V., Burenin, R. A., et al. 2009, ApJ, 692, 1060

Vikhlinin, A., Markevitch, M., Murray, S. S., et al. 2005, ApJ, 628, 655

Warren, M. S., Abazajian, K., Holz, D. E., \& Teodoro, L. 2006, ApJ, 646, 881

White, M., \& Vale, C. 2004, Astropart. Phys., 22, 19

Wu, X.-P., \& Mao, S. 1996, ApJ, 463, 404

Yoshida, N., Springel, V., White, S. D. M., \& Tormen, G. 2000, ApJ, 544, L87

Yoshida, N., Sheth, R., \& Diaferio, A. 2001, MNRAS, 328, 669

Zhang, Y.-Y., Finoguenov, A., Böhringer, H., et al. 2008, A\&A, 482, 451

Zhang, Y.-Y., Okabe, N., Finoguenov, A., et al. 2010, ApJ, 711, 1033 